\title{
Hadronic top-quark pair-production with one jet and parton showering
}

\author{
Simone Alioli, ${ }^{a}$ Sven-Olaf Moch $^{a}$ and Peter Uwer ${ }^{b}$ \\ ${ }^{a}$ Deutsches Elektronen-Synchrotron DESY, \\ Platanenallee 6, 15738 Zeuthen, Germany \\ ${ }^{b}$ Institut für Physik, Humboldt-Universität zu Berlin, \\ Newtonstr. 15, 12489 Berlin, Germany \\ E-mail: simone.alioli@desy.de, sven-olaf.moch@desy.de, \\ Peter.Uwer@physik.hu-berlin.de
}

\begin{abstract}
We present a calculation of heavy-flavor production in hadronic collisions in association with one jet matched to parton shower Monte Carlo programs at next-toleading order in perturbative QCD. Top-quark decays are included and spin correlations in the decay products are taken into account. The calculation builds on existing results for the radiative corrections to heavy-quark plus one jet production and uses the POWHEG BOX for the interface to the parton shower programs PYTHIA or HERWIG. A broad phenomenological study for the Large Hadron Collider and the Tevatron is presented. In particular we study - as one important sample application - the impact of the parton shower on the top-quark charge asymmetry.
\end{abstract}

KEYWords: QCD Phenomenology

ARXIV EPRINT: 1110.5251 


\section{Contents}

1 Introduction $\quad 1$

2 Implementation $\quad 2$

2.1 Checks 5

3 Results 5

$\begin{array}{ll}3.1 \text { Tevatron } & 7\end{array}$

3.1.1 Top-quark forward-backward charge asymmetry 11

$\begin{array}{lll}3.2 & \text { LHC } & 16\end{array}$

$\begin{array}{lll}3.2 .1 & \text { Top-quark charge asymmetry } & 20\end{array}$

$\begin{array}{lll}3.3 & \text { Top-quark decay } & 23\end{array}$

4 Conclusions $\quad 30$

$\begin{array}{ll}\text { A Folding } & 31\end{array}$

\section{Introduction}

The Large Hadron Collider (LHC) and the Tevatron provide a unique experimental environment for top-quark physics. Already with the currently accumulated luminosity at both colliders, but even more so with the anticipated high statistics data in the LHC $7 \mathrm{TeV}$ run, the prospects for precision studies of top-quarks are excellent. Precise experimental measurements for top-quark production demand theoretical predictions with comparable precision. Within Quantum Chromodynamics (QCD) this requires the knowledge of the hard scattering process beyond the leading order (LO) in perturbation theory. Predictions for the hadroproduction of top-quark pairs, i.e., $p p \rightarrow t \bar{t}$, are available at next-to-leading order (NLO) since long [1-4], and can be improved further thanks to resummation or the use of approximate next-to-next-to-leading order (NNLO) results, see e.g. [5, 6]. The NLO QCD corrections for the processes $p p \rightarrow t \bar{t}+$ jet $[7,8]$ and $p p \rightarrow t \bar{t}+2$ jets $[9,10]$ are more recent achievements.

For the direct comparison with experimental data, however, e.g., in order to model experimental acceptances, it is important to provide also theory predictions for the production of fully exclusive events. Due to the inherent limitations of the fixed-order approaches, in the past these were simulated by shower Monte Carlo (SMC) programs, which were accurate to LO only. Nowadays, much better theoretical precision can be reached by merging NLO computations with parton showers. The merging method has been pioneered by MC@NLO [11]. As an attractive feature the approach combines the accuracy of exact hard matrix elements for the large angle scattering including the radiative corrections to first 
order in the strong coupling constant $\alpha_{\mathrm{S}}$ with the soft and collinear emission described by the parton shower. The former ingredient usually displays a reduced sensitivity with respect to variations of the renormalization and factorization scales $\mu_{\mathrm{R}}$ and $\mu_{\mathrm{F}}$, while the latter accounts for the correct Sudakov suppression of collinear and soft emissions. The matching between the regions of hard and of soft and collinear emissions is smooth.

The follow-up development has seen an extension of the merging procedure by means of a method, dubbed POWHEG [12], which is independent of the parton SMC generator used. Moreover, the POWHEG method allows for the generation of positive weighted events only and thus for a very efficient event generation. The POWHEG BOX [13] now provides a general framework for implementing NLO calculations in SMC programs (see also [14] for progress towards further automation in MC@NLO). Processes involving the hadronic production of top-quarks have been subject to these improvements from very early on. Top-quark pair-production and single-top production processes have been implemented in MC@NLO $[15,16]$ and POWHEG [17-19] since quite some time and are now widely used by experimental collaborations.

In this article, we are concerned with $t \bar{t}$ pair-production in association with one hard jet, because a large fraction of inclusive $t \bar{t}$-events does actually contain one or even more additional jets. Moreover, due to the larger phase space, the relative importance of data samples with $t \bar{t}+$ jets increases at the LHC with respect to the Tevatron. Top-quark pairproduction associated with jets is also an important background to Higgs boson production in vector boson fusion and for many signals of new physics, e.g., those motivated by supersymmetry. For the process $t \bar{t}+$ jet we are merging the NLO QCD corrections $[7,8]$ (see [20] for the impact of top-quark decays) with the parton SMC HERWIG [21] and PYTHIA [22, 23] using the POWHEG BOX. An independent implementation of this process using the results of HELAC-NLO [9, 24] has been reported recently in ref. [25]. In this work we also include spin-correlation effects in the produced events.

The outline is a follows. In section 2 we discuss the implementation details in the POWHEG BOX, which are specific to the process $t \bar{t}+$ jet. We briefly describe the validation procedure and carefully examine details of the event generation in POWHEG. In section 3 we present a phenomenological analysis for the Tevatron with $\sqrt{s}=1.96 \mathrm{TeV}$ center-of-mass energy (section 3.1) and the LHC for $\sqrt{s}=7 \mathrm{TeV}$ (section 3.2). In case of the Tevatron we investigate the impact of the parton shower on the forward-backward charge asymmetry which is currently measured at the Tevatron for the inclusive sample. For the LHC the impact on the charge asymmetry visible in the rapidity distribution is analyzed. Furthermore we discuss the inclusion of the top-quark decay and its impact on differential distributions of the decay products. Our conclusions and our outlook on future developments are given in section 4 .

\section{Implementation}

The POWHEG BOX [13] provides a well-defined framework for implementing general NLO calculations in parton SMC programs. To that end, it requires as input particular information about the individual components of the NLO calculation under consideration, i.e., 
about the Born process, its virtual radiative corrections and the real emission contributions. Combination of the latter two and cancellation of the emerging soft and collinear singularities also requires the definition of a subtraction scheme. Within the POWHEG BOX the so-called Frixione-Kunszt-Signer (FKS) subtraction [26] is the method of choice.

For the reaction $t \bar{t}+$ jet we are building on the computation of $[7,8]$ for the QCD corrections to NLO, which provides us with all necessary details. The list of all flavor structures of the partonic Born and the real emission processes can be obtained from generic matrix elements by considering all possible crossings of light particles into the initial state. In Born approximation these are given by

$$
0 \rightarrow t \bar{t} g g g, \quad 0 \rightarrow t \bar{t} q \bar{q} g
$$

and for the real corrections by

$$
0 \rightarrow t \bar{t} g g g g, \quad 0 \rightarrow t \bar{t} q \bar{q} g g, \quad 0 \rightarrow t \bar{t} q \bar{q} q^{\prime} \bar{q}^{\prime}, \quad 0 \rightarrow t \bar{t} q \bar{q} q \bar{q},
$$

with $q, q^{\prime}=\{u, d, s, c, b\}$ and $q \neq q^{\prime}$.

We use MadGraph [27] for the computation of all Born squared amplitudes from eq. (2.1) and real emission matrix elements squared from eq. (2.2). Likewise, the color correlated Born squared amplitudes and the helicity correlated ones, both being necessary ingredients of NLO calculations employing a subtraction method, have been obtained by properly modifying the routines generated by MadGraph. Cross checks have been performed with AutoDipole [28]. Moreover, factorization in the soft and collinear limits has been explicitly checked in double and quadruple precision.

The finite part of the virtual corrections for $t \bar{t}+$ jet is extracted from a C++ library of results $[7,8]$, which is compliant with the interface to parton SMC programs proposed in [29]. In brief, the virtual amplitudes and loop diagrams associated with eq. (2.1) are all generated analytically and, after manipulation with computer algebra programs, automatically translated into $\mathrm{C}++$ code. The reduction of tensor integrals up to five-point functions displays good numerical stability. Finally, the calculation results in a decomposition of all loop diagrams according to helicity and color structure times scalar functions depending on the external momenta only. The infrared (IR)-finite scalar integrals are evaluated using the FF package $[30,31]$ and an efficient caching system is applied to speed-up the entire computation. The current implementation needs approximately $36 \mathrm{~ms}$ CPU time on an Intel Xeon processor with $3.00 \mathrm{GHz}$ for each evaluation of the $\tilde{B}$ function (see eq. 4.5 of [13]) at a single phase space point, summing over all contributing subprocesses. About half of that time is spent on the computation of the virtual corrections.

With the input described so far, our POWHEG BOX implementation can be used to generate hadronic events accurate to NLO. There is, however, an important technical issue related to multi-leg processes which are interfaced to a parton shower: Reactions such as $t \bar{t}+$ parton possess soft and collinear divergences already at LO. Since the POWHEG method relies on generating all events starting from a momentum configuration of the underlying Born process, a generation cut, $p_{\mathrm{T}}^{\text {gen }}$, needs to be placed on the transverse momentum of the final-state partons, a procedure well-known from standard SMC generators, see e.g., the discussion in [32]. 


\begin{tabular}{|ccccc|}
\hline & $p_{\mathrm{T}}^{\text {gen }}[\mathrm{GeV}]$ & $p_{\mathrm{T}}^{\text {supp }}[\mathrm{GeV}]$ & $p_{\mathrm{T}}^{\text {an }}[\mathrm{GeV}]$ & $\sigma^{\mathrm{NLO}}[\mathrm{pb}]$ \\
\hline & 0 & 20 & 20 & $1.793 \pm 0.002$ \\
TEV $1.96 \mathrm{TeV}$ & 2 & 0 & 20 & $1.790 \pm 0.001$ \\
& 2 & 20 & 20 & $1.791 \pm 0.002$ \\
& 2 & 200 & 20 & $1.793 \pm 0.002$ \\
& 5 & 0 & 20 & $1.782 \pm 0.001$ \\
& 5 & 20 & 20 & $1.785 \pm 0.001$ \\
\hline \multirow{4}{*}{ LHC $7 \mathrm{TeV}$} & 0 & 400 & 50 & $52.6 \pm 0.5$ \\
& 5 & 400 & 50 & $52.7 \pm 0.5$ \\
& 5 & 100 & 50 & $53.1 \pm 0.2$ \\
& 10 & 0 & 50 & $52.9 \pm 0.4$ \\
LHC $14 \mathrm{TeV}$ & 10 & 400 & 50 & $52.5 \pm 0.1$ \\
& 0 & 0 & 50 & $52.6 \pm 0.4$ \\
\hline & 5 & 400 & 50 & $379.8 \pm 1.6$ \\
& 5 & 400 & 50 & $376.1 \pm 0.2$ \\
& 15 & 50 & $377.2 \pm 1.6$ \\
\hline
\end{tabular}

Table 1. The dependence of the NLO cross section at Tevatron and LHC on the generation cut $p_{\mathrm{T}}^{\text {gen }}$ and on the Born suppression factor $p_{\mathrm{T}}^{\text {supp }}$ for $\mu_{\mathrm{R}}=\mu_{\mathrm{F}}=m_{t}=174 \mathrm{GeV}$ and CTEQ6M PDFs. Jets are reconstructed by the inclusive $k_{\mathrm{T}}$-algorithm with $R=1$.

This cut for the event generation, which in practice takes values e.g., $p_{\mathrm{T}}^{\text {gen }} \approx 1 \mathrm{GeV}$, is unphysical and has to be always much smaller than the analysis cut on the transverse momentum, $p_{\mathrm{T}}^{\text {an }}$, employed in the definition of the additional jet associated with the $t \bar{t}$ pair. The condition $p_{\mathrm{T}}^{\text {gen }} \leq p_{\mathrm{T}}^{\text {an }}$ is necessary though not sufficient to provide a suitable sample of unweighted events, because the parton shower can in fact increase the transverse momentum in an event generated at Born level with a given $p_{\mathrm{T}} \leq p_{\mathrm{T}}^{\text {gen }}$. This may result in a different number of events that would have passed the analysis cut if the generation cut were different. Therefore, it is essential to show that the actual dependence on $p_{\mathrm{T}}^{\text {gen }}$ is negligible for a given fixed analysis cut $p_{\mathrm{T}}^{\text {an }}$. We have carefully investigated this issue, reporting some examples in table 1 , which shows the independence of the cross section on the generation cut $p_{\mathrm{T}}^{\text {gen }}$ for reasonable values of the cut. In particular, this is satisfied for $p_{\mathrm{T}}^{\text {gen }}$ smaller than $p_{\mathrm{T}}^{\text {an }} / 4$. Table 1 provides also a strong cross check of existing results in [25].

Also a second option for dealing with processes with soft and collinear divergences at Born level is available by generating weighted rather than unweighted events and, thereby suppressing the divergences. The POWHEG BOX uses a suppressed cross section $\bar{B}_{\text {supp }}$ for the generation of the underlying Born configurations [32],

$$
\bar{B}_{\text {supp }}=\bar{B} \times F\left(p_{\mathrm{T}}\right),
$$

where $p_{\mathrm{T}}$ is a measure of the hardness of the extra emission, $\bar{B}$ denotes the inclusive NLO cross section at fixed underlying Born variables (see eq. 4.2 of [13]), and $F\left(p_{\mathrm{T}}\right)$ has to be chosen such that it ensures finiteness of $\bar{B}_{\text {supp }}$ in the limit $p_{\mathrm{T}} \rightarrow 0$. Thus, $\bar{B}_{\text {supp }}$ becomes integrable and, of course, the generated events need to be weighted by $1 / F\left(p_{\mathrm{T}}\right)$. The 
POWHEG BOX implementation foresees the choice

$$
F\left(p_{\mathrm{T}}\right)=\left(\frac{p_{\mathrm{T}}^{2}}{p_{\mathrm{T}}^{2}+\left(p_{\mathrm{T}}^{\text {supp }}\right)^{2}}\right)^{n},
$$

where the variable $p_{\mathrm{T}}^{\text {supp }}$ controls the extent of the suppression ${ }^{1}$ and $n$ is process specific, $n=2$ for $t \bar{t}+$ jet. In our event generation we have also applied the Born suppression factor of eq. (2.4) and checked its implementation for different $p_{\mathrm{T}}^{\text {supp }}$ values with very good numerical accuracy, as shown in table 1.

\subsection{Checks}

The current implementation has been exposed to a large number of checks. These include, first of all, comparisons for a sizable number of differential distributions to NLO with the available fixed order results. We have used the settings of $[7,8]$, i.e., the renormalization and factorization scales $\mu_{R}=\mu_{F}=m_{t}=174 \mathrm{GeV}$, the analysis cut of $p_{\mathrm{T}}>20 \mathrm{GeV}$ and $p_{\mathrm{T}}>50 \mathrm{GeV}$ for the Tevatron and LHC configuration, respectively, the parton distribution function (PDF) set CTEQ6M [33], the jet algorithm of [34] (inclusive- $k_{T}$ ) with $R=1$ and we have assumed here the top-quarks to be always tagged and thus excluded from jet reconstruction. Perfect agreement with $[7,8]$ has been found typically at the per-mille level. Also note that the computation of $[7,8]$ has employed dipole subtraction with massive partons [35] for the cancellation of the soft and collinear divergences between the real and virtual contributions. As mentioned, the POWHEG BOX relies on FKS subtraction [26] and, of course, the results for physical cross sections at NLO must be independent of the chosen scheme, which constitutes another strong check of the current implementation.

The other aspect which demands careful checking is the parton shower. It adds allorder perturbative corrections, though to leading logarithmic accuracy only, and the POWHEG approach offers one particular choice of how these corrections beyond NLO are included. Since the merging with parton showers modifies the QCD predictions, sometimes even for inclusive quantities, one should check during the validation process that these modifications reflect real physics effects and moreover, are compatible with (un)known higher-order corrections. To that end, we have also compared the NLO fixed order and the POWHEG predictions after the first emission, i.e., at the level they are written to the Les Houches event file [36] (LHEF from now on), for suitable distributions in order to assess similarities and differences between the results. Of particular interest in this procedure are those observables, which display great sensitivity to the parton shower effects. Some results will be shown below in section 3 .

\section{Results}

Throughout this section we present our findings. In comparing to the fixed order NLO results we are using the parton shower programs HERWIG [21] (version 6.5.20) and PYTHIA [22,

\footnotetext{
${ }^{1}$ In the POWHEG BOX the value of $p_{\mathrm{T}}^{\text {supp }}$ can be assigned at run time trough the bornsuppfact entry of the input card.
} 
23] (version 6.4.25), with the default setting of parameters. Moreover, in order to highlight the shower effects and to disentangle them from the underlying event (UE) or multiple particle interactions (MPI), we have generally switched off these features in the SMC programs. Of course MPI and UE, together with the usage of the appropriate SMC tuning, will play an important rôle when comparing POWHEG predictions with data. For the kind of study we are interested in, we have decided not to include them in the following plots and we have concentrated on presenting distributions which are not extremely sensitive to these effects, where possible. The one exception here is the discussion of the charge asymmetries, in sections 3.1.1 and 3.2.1. There, we will also consider the predictions obtained including also MPI and UE in our comparisons in order to asses the stability of our results with respect to these effects.

We have produced different samples of events, to study both, the Tevatron and LHC collider configurations. Each sample contains 20M positive and negative weighted events, produced without folding (see appendix A) and with a generation cut $p_{\mathrm{T}}^{\text {gen }}=2 \mathrm{GeV}$ and $5 \mathrm{GeV}$, respectively, for the Tevatron and the LHC. As already mentioned, the jet reconstruction cut in the analysis has been assumed to be $p_{\mathrm{T}}>20 \mathrm{GeV}$ and $50 \mathrm{GeV}$. We also make use of the bornsuppfact option of the POWHEG BOX to damp the low transverse momentum regions in the cross section, artificially increasing the probability of a hard jet. For this reason, the events have a weight which is the inverse of eq. (2.4). The choices $p_{\mathrm{T}}^{\text {supp }}=20(100) \mathrm{GeV}$ for the Tevatron (LHC) were adopted.

For the sake of comparisons and, in order to keep the analysis routine rather insensitive to possible contamination in the jet reconstruction procedure, we always force the semileptonic decay of the (anti-)top-quark when interfacing to a SMC program in the following. Also, as already done for the NLO comparisons of section 2, we will always use the jet algorithm of [34] (inclusive- $k_{T}$ ) with $R=1$ and the $E_{T}$-recombination scheme. ${ }^{2}$ In addition, after interfacing to a SMC program and performing the hadronization stage, we always exclude from the jet list those jets which happen to contain a $b$-flavored hadron, whose origin can be traced back to the (anti-)top-quark decay. ${ }^{3}$.

As we focus entirely on the effect of the parton SMC and the NLO merging, our phenomenological analysis in sections 3.1 and 3.2 follows $[7,8]$, fixes the renormalization and factorization scales $\mu_{R}=\mu_{F}=m_{t}=174 \mathrm{GeV}$, and uses the PDF set CTEQ6M [33]. Studies of the theoretical uncertainty of the cross section for $p p \rightarrow t \bar{t}+$ jet due to corrections of higher orders (beyond NLO in QCD) have already been conducted in $[7,8]$ with standard means, i.e., determining the variations for the scale choices of $\mu_{\mathrm{R}}=\mu_{\mathrm{F}}=m_{t} / 2$ and $\mu_{\mathrm{R}}=\mu_{\mathrm{F}}=2 m_{t}$. In table 2 we report results for the Tevatron $1.96 \mathrm{TeV}$ as well as for the $7 \mathrm{TeV}$ and $14 \mathrm{TeV}$ LHC configurations allowing for the independent variations of $\mu_{\mathrm{R}}$ and

\footnotetext{
${ }^{2}$ Comparisons have been done with other jet clustering algorithms, e.g., the anti- $k_{T}$ jet algorithm [37] with $p$-scheme recombination, for different values of the parameter $R$, without significantly changing the general conclusions drawn here.

${ }^{3}$ This exclusion is applied also when the top-quark decay is performed by POWHEG itself and bottomquarks coming from top-quarks can be present already at the LHEF stage, as it will be the case in Sec 3.3. Other bottom-quarks present at partonic level (or B hadron not originating from a (anti-)top-quark) are instead always consistently considered as (originating from) light partons and therefore included in the light jets count.
} 


\begin{tabular}{|ccc|}
\hline & $p_{\mathrm{T}}^{\text {an }}[\mathrm{GeV}]$ & $\sigma_{\mathrm{NLO}}[\mathrm{pb}]$ \\
\hline TEV $1.96 \mathrm{TeV}$ & 20 & $1.791(2)_{-0.318}^{+0.160}$ \\
LHC $7 \mathrm{TeV}$ & 50 & $53.1(2)_{-8.9}^{+4.1}$ \\
LHC $14 \mathrm{TeV}$ & 50 & $376.1(2)_{-45.4}^{+20.1}$ \\
\hline
\end{tabular}

Table 2. The dependence of the NLO cross section at Tevatron and LHC on the renormalization $\mu_{\mathrm{R}}$ and factorization scales $\mu_{\mathrm{F}}$. The envelope has been constructed by considering independent variations of $\mu_{\mathrm{R}}$ and $\mu_{\mathrm{F}}$ around $m_{t}=174 \mathrm{GeV}$, by a factor of two in both directions. Combinations resulting in ratios of $\mu_{\mathrm{R}} / \mu_{\mathrm{F}}$ larger than 2 or smaller than $1 / 2$ have been excluded. Jets are reconstructed by the inclusive $k_{\mathrm{T}}$-algorithm with $R=1$ and CTEQ6M PDFs are adopted.

$\mu_{\mathrm{F}}$ of factors $m_{t} / 2$ and $2 m_{t}$ and, at the same time, excluding relative ratios of $\mu_{\mathrm{R}} / \mu_{\mathrm{F}}$ larger than 2 or smaller than $1 / 2$. We find a remarkable small variation, typically below $10 \%$. On the basis of the scale variations uncertainty one can thus conclude that higher order effects should be small.

The other main source of theoretical uncertainties, related to the non-perturbative parameters such as the PDFs and the associated value of the strong coupling constant $\alpha_{\mathrm{S}}\left(M_{Z}\right)$, have repeatedly been addressed in the literature (see e.g., [6]) using modern PDF sets available to NNLO [38-40]. For a more recent study concerning the combined uncertainty due to propagated PDF uncertainties and uncertainties in $\alpha_{\mathrm{S}}\left(M_{Z}\right)$ we refer the interested reader to the results discussed in [41]. The top-quark mass $m_{t}$ is always taken as a pole mass, and we refer to [42] for a discussion of the running mass in the $\overline{\mathrm{MS}}$-scheme.

\subsection{Tevatron}

We will begin our studies with the Tevatron $(\sqrt{s}=1.96 \mathrm{TeV})$, which has provided us already with several measurements of differential distributions. These are, in detail, the first measurement of the $t \bar{t}$ cross section $d \sigma / d m_{t \bar{t}}$, differential in $t \bar{t}$-pair invariant mass $m_{t \bar{t}}$, see [43], the dependence of the $t \bar{t}$ production cross section on the transverse momentum of the top-quark [44] and most prominently, the measurements of the forward-backward charge asymmetry in top-quark pair-production $[45,46]$ along with its dependence on the $t \bar{t}$-pair invariant mass $m_{t \bar{t}}[47,48]$.

In all the plots throughout this article no acceptance cuts are imposed, other than those necessary to define the hard jet. The lines appearing the legenda are labeled according their origin: NLO for the fixed order computation, LHEF for the results after the POWHEG hardest emission without any showering and PWG+HER, PWG+PYT for the results obtained by generating the hardest emission with the POWHEG method and then performing the remaining shower and hadronization stages with the HERWIG or PYTHIA programs, respectively. The lower inset in each plot shows the relative difference with respect to the first entry in the legend, which is usually depicted as a solid black line in the upper inset. The black dots in the lower inset stand instead as a reference for the zero line. The Monte Carlo integration errors are shown as vertical bars. 

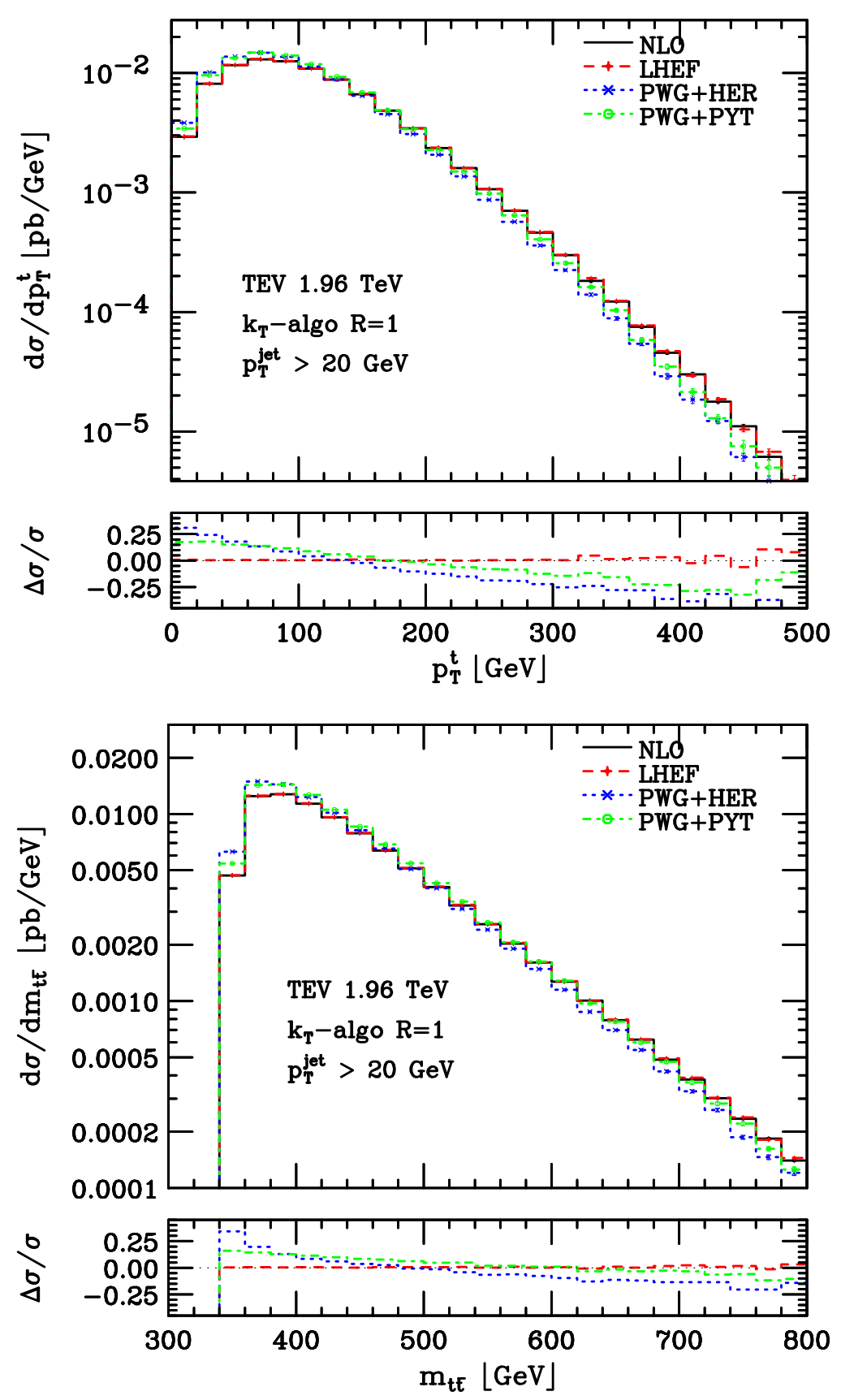

Figure 1. The differential cross sections as function of the transverse momentum $p_{\mathrm{T}}^{t}$ (upper panel) and of the $t \bar{t}$-pair invariant mass $m_{t \bar{t}}$ (lower panel), at the Tevatron $(\sqrt{s}=1.96 \mathrm{TeV})$.

In figure 1, we plot the differential cross section as a function of the transverse momentum $p_{\mathrm{T}}^{t}$ of the top-quark (upper panel) and of the the $t \bar{t}$-pair invariant mass $m_{t \bar{t}}$ (lower panel). A first comment can be made upon the expected good agreement between the NLO results and the LHEF ones for such inclusive quantities. The effect of the shower does not change considerably these results. Only at the very low and high ends we observe a significant change which is however still below $30 \%$. 

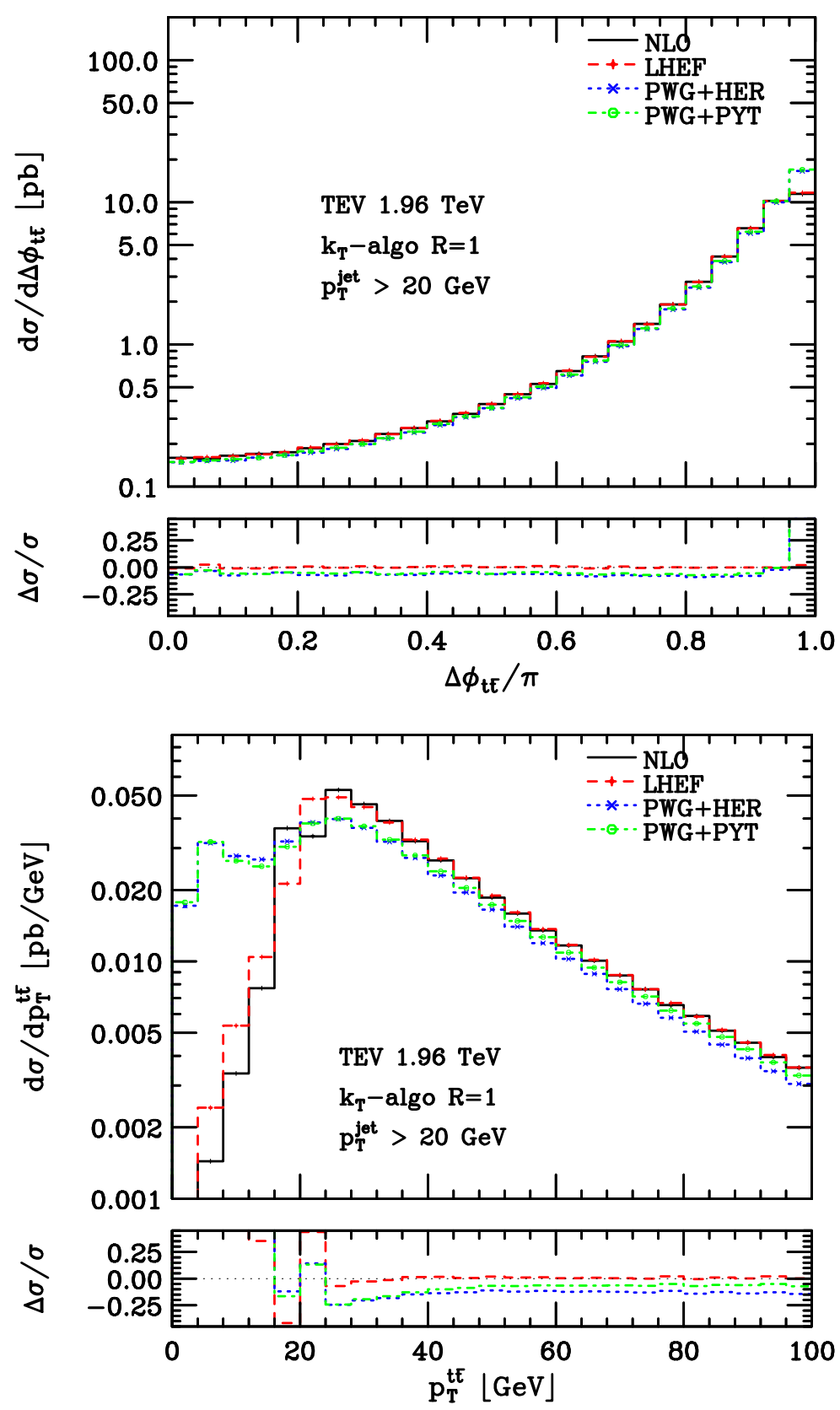

Figure 2. The differential cross sections as function of the $t \bar{t}$-pair azimuthal separation $\Delta \phi_{t \bar{t}}$ and transverse momentum $p_{\mathrm{T}}^{t \bar{t}}$, at the Tevatron $(\sqrt{s}=1.96 \mathrm{TeV})$.

Similar conclusions can also be drawn looking at the upper panel of figure 2, where we plot the differential cross sections $d \sigma / d \Delta \phi_{t \bar{t}}$ as a function of the azimuthal separation between the top- and anti-top-quarks. In particular, this last observable is very stable with respect to the inclusion of the parton shower. With exception of the last bin, we observe corrections of a few percent only. The understanding is that the parton shower is not able to produce a significant change of the relative directions of the two outgoing 
heavy quarks. In the lower panel instead, we show the differential cross sections $d \sigma / d p_{\mathrm{T}}^{t \bar{t}}$ as a function of the transverse momentum of the $t \bar{t}$-pair. Shower effects at low $p_{\mathrm{T}}^{t \bar{t}}$ values are now clearly visible. Furthermore, by inspecting the lower inset in the plot, showing the relative difference with NLO predictions, it is also possible to see a hint of the instabilities that may arise in the fixed order calculation around the $p_{\mathrm{T}}=20 \mathrm{GeV}$ jet cut.

This behaviour is easily explained considering that the region below the cut may be populated only when more than one hard jet is resolved, thus allowing for an imbalance between the hardest jet and the $t \bar{t}$-pair. Indeed, for the 3 -partons $t \bar{t} j$ configuration, $p_{\mathrm{T}}^{t \bar{t}}=p_{\mathrm{T}}^{j 1}$ by momentum conservation. This effectively makes the $d \sigma / d p_{\mathrm{T}}^{t \bar{t}}$ distribution for $p_{\mathrm{T}}^{t \bar{t}}$ lower than the jet $p_{\mathrm{T}}$ cut a LO quantity, starting at $\mathcal{O}\left(\alpha_{\mathrm{S}}^{4}\right)$. We have verified that the instability gets worse by reducing the bin size and that neither the LHEF nor the showered results show a similar behaviour. The same feature will also be present in LHC predictions, around the $p_{\mathrm{T}}=50 \mathrm{GeV}$ jet cut, as we will show in the lower panel of figure 7. Such instabilities are well known to arise at any fixed order of the perturbative expansion whenever the observable under consideration has a non-smooth behaviour inside the physical region or whenever the phase space boundary for a certain number of partons lies inside that for a larger number [49]. In our case the LO $d \sigma / p_{\mathrm{T}}^{t \bar{t}}$ distribution is discontinuous at the jet $p_{\mathrm{T}}^{\text {cut }}$ boundary, since the phase space for 3 partons does not allow $p_{\mathrm{T}}^{t \bar{t}}=p_{\mathrm{T}}^{j 1}<p_{\mathrm{T}}^{\text {cut }}$, while it is different from zero at $p_{\mathrm{T}}^{t \bar{t}} \geq p_{\mathrm{T}}^{\text {cut }}$. It should be stressed that despite these effects are due to unbalanced cancellation of higher order soft/collinear divergences at the critical point(s), they are integrable in any finite neighborhood. In ref. [49] it was also demonstrated that the all-order resummation of soft divergences restores the infrared finiteness of the predictions also at the critical point by the appearance of continuous and infinitely differentiable structures, that were dubbed Sudakov shoulders. We have indeed verified that the resummation implicit in the POWHEG approach does reproduce these structures, as can be observed in the figures.

From figure 3 on we investigate the jet structure of the process. To that end, in figure 3 , we display the differential cross sections $d \sigma / d p_{\mathrm{T}}^{j_{1 s t}}$ (upper panel) and $d \sigma / d y_{j}$ st (lower panel) as function of the transverse momentum and rapidity of the hardest jet. Here we notice a moderate shape distortion and a different normalization in going from parton level to hadronic events. Both these effects can be understood by considering that the jet clustering procedure is significantly affected when going from a low multiplicity sample to a fully hadronized event. This is also reflected in the number of resolved jets above the minimum $p_{\mathrm{T}}$ cut and in their kinematics.

In the upper panel of figure 4 we show the azimuthal difference $\Delta \phi_{t \bar{t}-j^{1 s t}}$ between the hardest jet and the $t \bar{t}$ system. $\Delta \phi_{t \bar{t}-j \text { 1st }}$ is very sensitive to shower effects, since at partonic level the hardest jet is constrained to reside in the opposite hemisphere w.r.t. the $t \bar{t}$-pair momentum. In the lower panel we plot the differential cross section $d \sigma / d p_{\mathrm{T}}^{t \bar{j} j_{1 s t}}$ as function of the transverse momentum of the system composed of the top-quark, the anti-top-quark and the hardest jet. This observable is of particular importance, as it is strongly correlated with the transverse momentum of the next-to-hardest jet. The latter, in turn, is the quantity where the Sudakov suppression effects should be most evident. Due to the jet reconstruction procedure, however, it turns out that those effects are best seen 
in the low $p_{\mathrm{T}}^{t \bar{t} j_{1 s t}}$ region. The disagreement in the high $p_{\mathrm{T}}^{t \bar{t} j_{1 s t}}$ tail between NLO and LHEF results, on the one side, and showered results, on the other, is instead due to showering and hadronization effects and is partly related to our choice of excluding the top-quark from jet reconstruction. A similar effect has also been observed (and explained) in singletop production (see section 4 of [50]) and we have verified that the same explanation can readily be applied here too, for the $t \bar{t} j_{1 \text { st }}$ system: At high $p_{\mathrm{T}}^{t \bar{t} j_{1 s t}}$ there exists an artificial imbalance due to many hadrons coming from the hardest parton which are not clustered together with the hardest jet. This creates an effective $p_{\mathrm{T}}$ for the system containing the hardest jet, giving rise to the harder $p_{\mathrm{T}}^{t \bar{t} j_{1 s t}}$ tail observed.

Finally, in figure 5 we plot the differential cross section as function of the transverse momentum $p_{\mathrm{T}}^{j_{2 n d}}$ and rapidity $y_{j \text { 2nd }}$ of the next-to-hardest jet. No high- $p_{\mathrm{T}}$ enhancement, or suppression, is observed in this case. However, large differences in the normalization of distributions are present when going from NLO results to LHEF and showered ones. This can again be explained with the different number of configurations with are resolved by the reconstruction algorithm as containing two hard jets.

\subsubsection{Top-quark forward-backward charge asymmetry}

In view of the measurements of the forward-backward asymmetry in top-quark pairproduction at the Tevatron [45-47] we have also evaluated the values of the $t \bar{t}$ rapidity asymmetries in $t \bar{t}+$ jet samples, at various stages of our simulations and with various acceptance cuts. The definitions for $A_{\mathrm{FB}}^{t}$ and $A_{\mathrm{FB}}^{t \bar{t}}$ used throughout this paper are

$$
A_{\mathrm{FB}}^{t}=\frac{1}{\sigma}\left(\int_{y_{t}>0} d \sigma-\int_{y_{t}<0} d \sigma\right), \quad A_{\mathrm{FB}}^{t \bar{t}}=\frac{1}{\sigma}\left(\int_{\Delta y_{t \bar{t}}>0} d \sigma-\int_{\Delta y_{t \bar{t}}<0} d \sigma\right),
$$

where $\Delta y_{t \bar{t}}=y_{t}-y_{\bar{t}}$. Due to the fact that the initial $p \bar{p}$ state at the Tevatron is a $\mathrm{CP}$ eigenstate and due to the absence of CP violating effects in QCD, one has $\frac{d \sigma}{d y_{t}}\left(y_{t}\right)=$ $\frac{d \sigma}{d y_{\bar{t}}}\left(-y_{\bar{t}}\right)$ and $A_{\mathrm{FB}}^{t \bar{t}}$ corresponds to $A_{\mathrm{FB}}^{t}$ in the $t \bar{t}$ rest frame. When moving to the laboratory frame, where a greater accuracy can be reached on the experimental determination of these quantities, $A_{\mathrm{FB}}^{t}$ gets smaller in magnitude than $A_{\mathrm{FB}}^{t \bar{t}}$, which in turn is a boost-invariant quantity, depending only on rapidity differences. We report both in table 3, expressed as percentage values to ease their readability, at various stages of our simulation. We have also included the predictions obtained with the HERWIG and PYTHIA showers supplemented with underlying-event and multi-parton interactions activities, respectively, referring to them as PWG+HER+UE and PWG+PYT+MPI. In case of PWG+PYT+MPI the Perugia 0 Tune [51] - $\operatorname{MSTP}(5)=320$ - has also been adopted.

For both asymmetries and shower models, we observe that the inclusion of the parton shower leads to a significant correction compared to the fixed order and LHEF predictions. For example, the results for total asymmetries are almost reduced by a factor of two by the parton-shower. A similar effect is also observed in presence of selection cuts on the rapidity or invariant mass of the heavy quark pair. This outcome extends the conclusions 

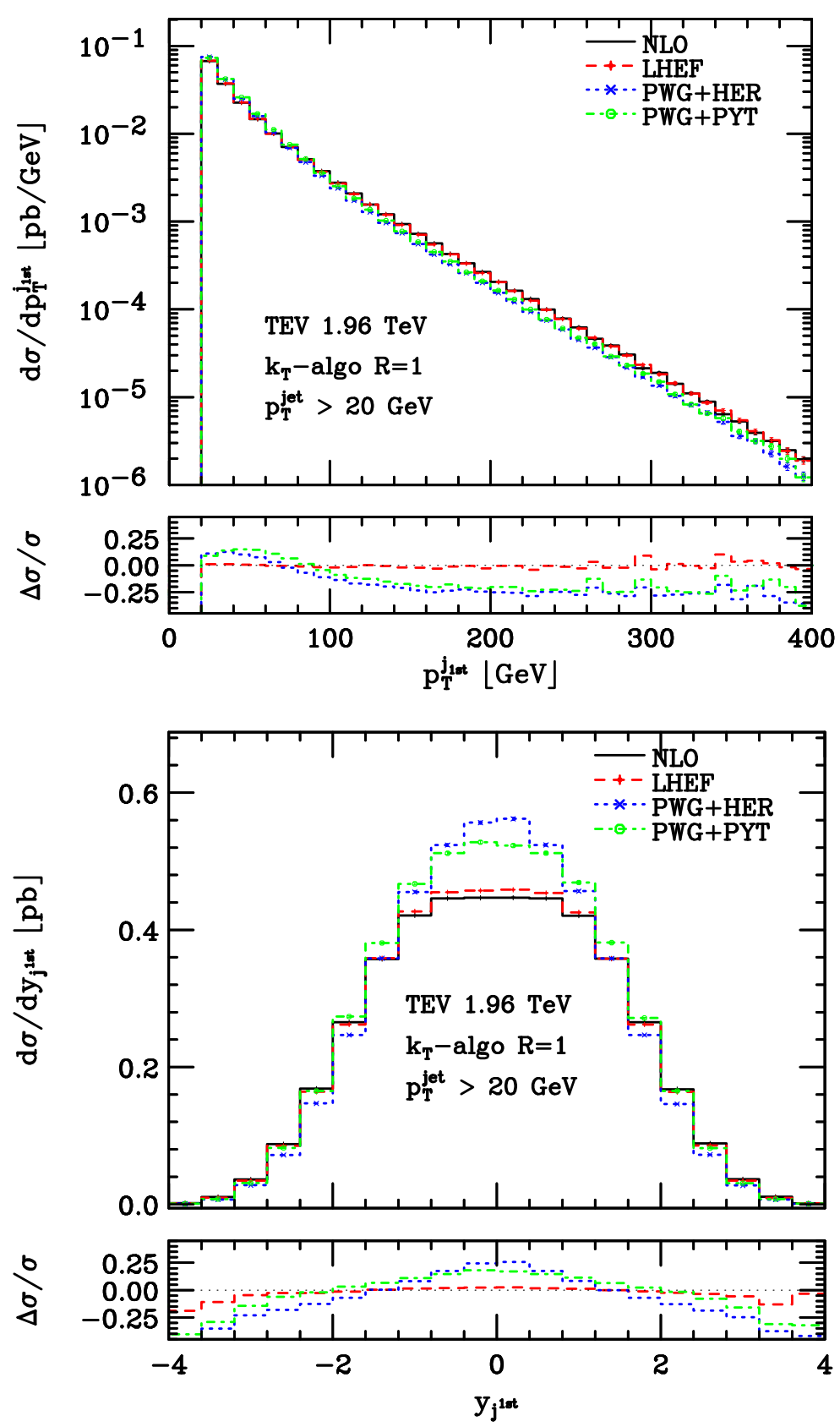

Figure 3. The differential cross sections as function of the hardest jet transverse momentum and rapidity at the Tevatron $(\sqrt{s}=1.96 \mathrm{TeV})$.

obtained by previous combined NLO and parton shower simulations [47] for the inclusive $t \bar{t}$ sample. ${ }^{4}$

Inspecting the results shown in table 3 more closely, we find that performing PYTHIA and HERWIG showers leads to similar predictions for $A_{\mathrm{FB}}^{t}$ : the values are fairly consistent

\footnotetext{
${ }^{4}$ See also [52] for higher-order QCD corrections to $A_{F B}$ and [10] for studies of the $t \bar{t}+2$ jet process in particular.
} 

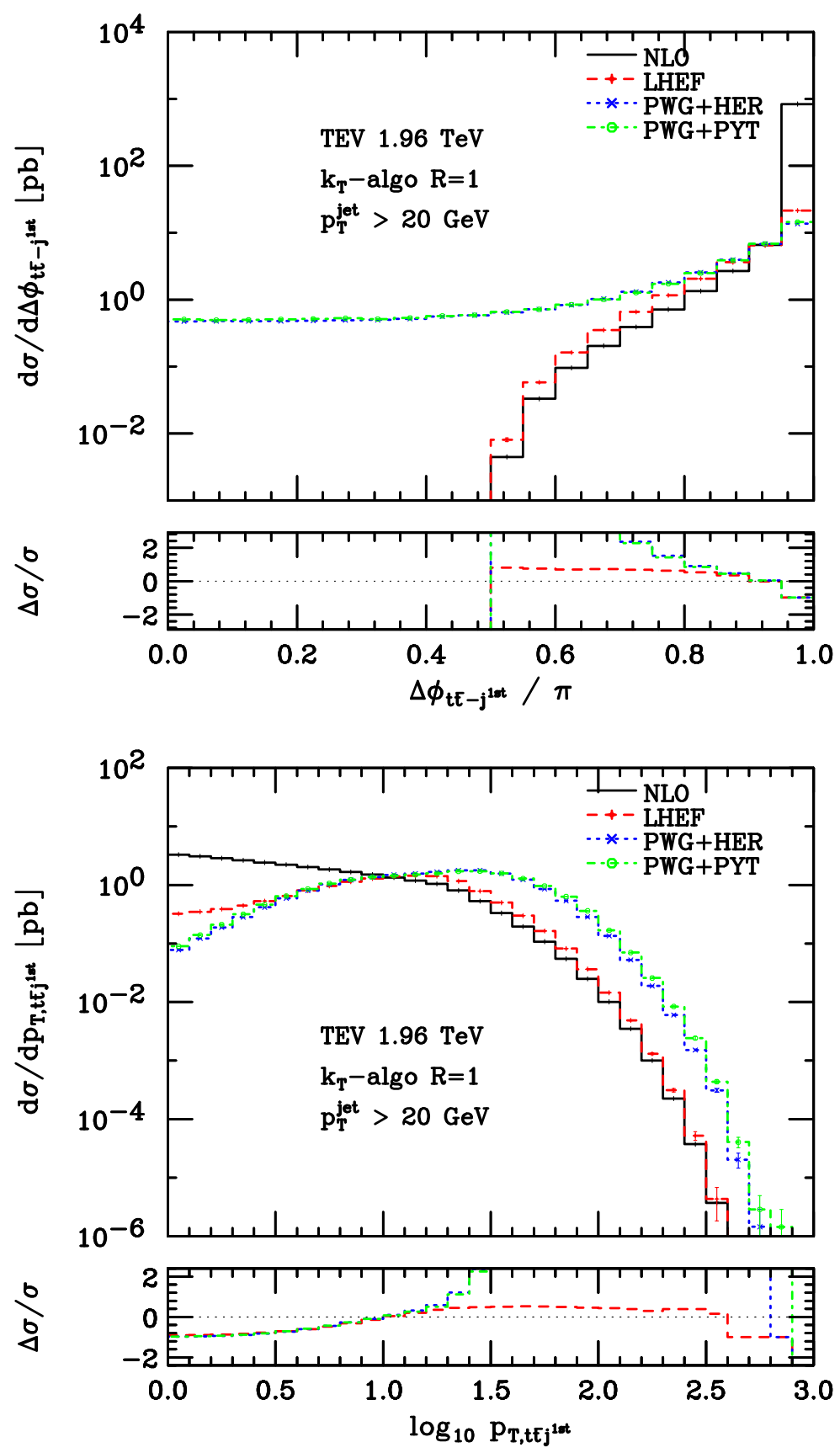

Figure 4. The differential cross sections as function of the azimuthal distance of the hardest jet from the $t \bar{t}$-pair (upper panel) and as a function of the transverse momentum of the system made by $t \bar{t}$-pair and the hardest jet (lower panel) at the Tevatron $(\sqrt{s}=1.96 \mathrm{TeV})$.

once the statistical uncertainties are taken into account. The agreement is instead much worse for $A_{\mathrm{FB}}^{t \bar{t}}$. The inclusion of underlying-event or multi-particle interaction does not dramatically change this picture, the interleaved shower evolution due to MPI and Perugia 0 Tune in PYTHIA results in a larger difference with respect to the default behaviour than the simpler UE model in HERWIG. 

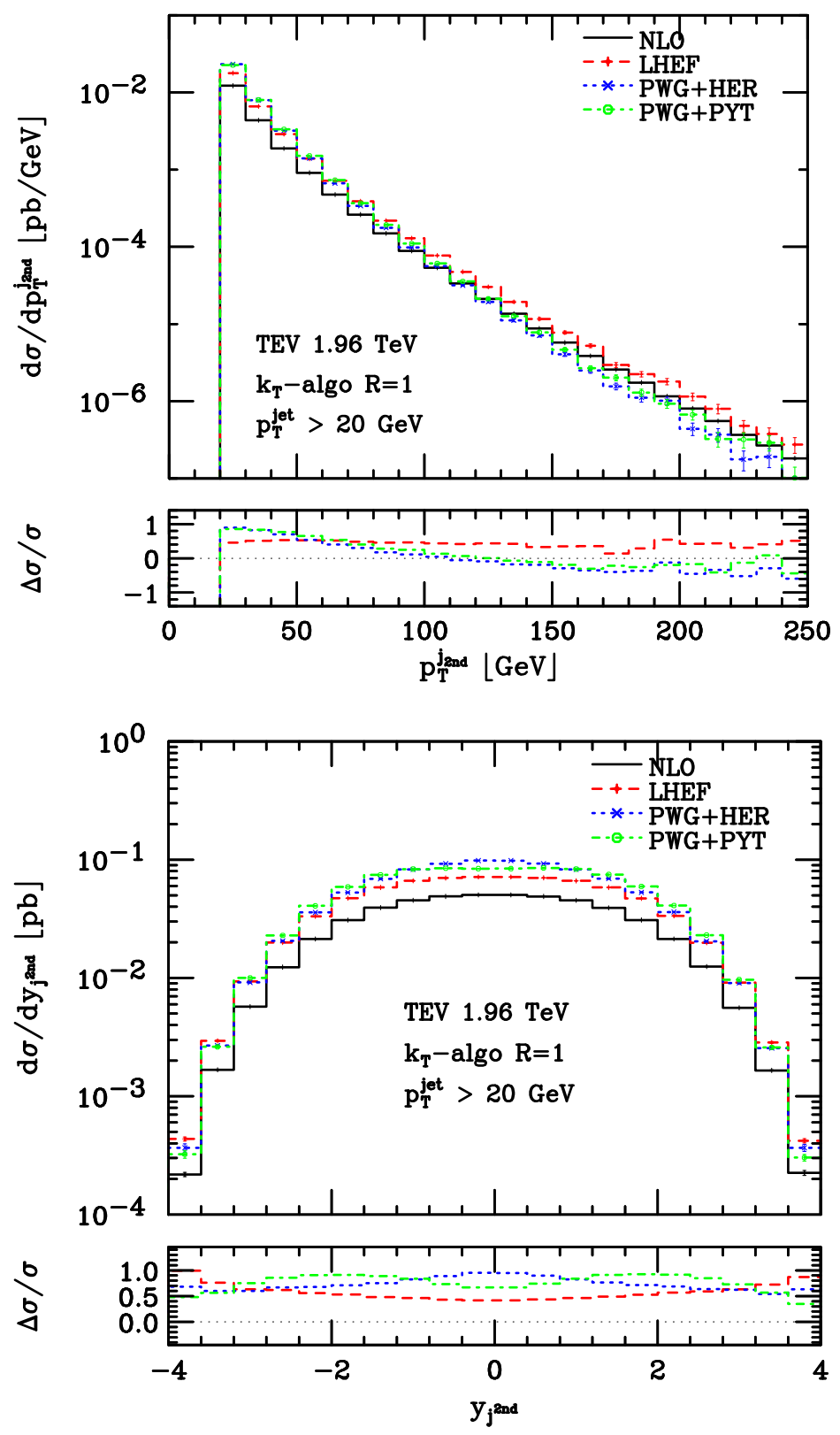

Figure 5. The differential cross sections as function of the next-to-hardest jet transverse momentum and rapidity at the Tevatron $(\sqrt{s}=1.96 \mathrm{TeV})$.

The dependence of $A_{\mathrm{FB}}$ on the transverse momentum of the $t \bar{t}$-pair, $p_{\mathrm{T}}^{t \bar{t}}$, has also recently been reported in [48]. In ref. [53], the authors have investigated this behaviour in presence of selection cuts on the $t \bar{t}$ pair invariant mass, including also EW corrections.

Since this observable only receives contributions starting at $\mathcal{O}\left(\alpha_{\mathrm{S}}^{3}\right)$, even for the $t \bar{t}$ inclusive sample - an extra jet is always required to have a non-zero $p_{\mathrm{T}}^{t \bar{t}}$ - our approach offers the possibility to evaluate it at NLO supplemented with shower effects for the first time. This should improve over the MC@NLO and POWHEG simulations for $t \bar{t}$ production, that are only leading-order/leading-logarithm accurate for $A_{\mathrm{FB}}$ when $p_{\mathrm{T}}^{t \bar{t}}>0$. 


\begin{tabular}{|c|c|c|c|c|c|c|}
\hline Tevatron $1.96 \mathrm{TeV}$ & NLO $[\%]$ & LHEF [\%] & PWG+HER [\%] & $\mathrm{PWG}+\mathrm{HER}+\mathrm{UE}[\%]$ & $\mathrm{PWG}+\mathrm{PYT}[\%]$ & $\mathrm{PWG}+\mathrm{PYT}+\mathrm{MPI}[\%]$ \\
\hline$A_{\mathrm{FB}}^{t}$ total & $-2.98 \pm 0.04$ & $-2.95 \pm 0.05$ & $-1.75 \pm 0.11$ & $-1.70 \pm 0.11$ & $-1.49 \pm 0.11$ & $-1.36 \pm 0.11$ \\
\hline$A_{\mathrm{FB}}^{t},\left|y_{t}\right|<1.0$ & $-2.60 \pm 0.04$ & $-2.55 \pm 0.05$ & $-1.51 \pm 0.12$ & $-1.53 \pm 0.11$ & $-1.31 \pm 0.12$ & $-1.22 \pm 0.12$ \\
\hline$A_{\mathrm{FB}}^{t},\left|y_{t}\right| \geq 1.0$ & $-6.38 \pm 0.19$ & $-6.51 \pm 0.15$ & $-3.79 \pm 0.35$ & $-3.15 \pm 0.34$ & $-2.99 \pm 0.35$ & $-2.58 \pm 0.34$ \\
\hline$A_{\mathrm{FB}}^{t},\left|m_{t \bar{t}}\right|<450 \mathrm{GeV}$ & $-1.90 \pm 0.06$ & $-1.80 \pm 0.06$ & $-1.24 \pm 0.14$ & $-1.24 \pm 0.13$ & $-0.81 \pm 0.14$ & $-1.00 \pm 0.14$ \\
\hline$A_{\mathrm{FB}}^{t},\left|m_{t \bar{t}}\right| \geq 450 \mathrm{GeV}$ & $-4.70 \pm 0.06$ & $-4.77 \pm 0.08$ & $-2.70 \pm 0.19$ & $-2.54 \pm 0.18$ & $-2.66 \pm 0.18$ & $-1.98 \pm 0.18$ \\
\hline$A_{\mathrm{FB}}^{t}, p_{\mathrm{T}}^{t \bar{t}} \geq 10 \mathrm{GeV}$ & $-2.95 \pm 0.04$ & $-2.93 \pm 0.05$ & $-2.64 \pm 0.06$ & $-2.59 \pm 0.06$ & $-2.58 \pm 0.06$ & $-2.39 \pm 0.06$ \\
\hline$A_{\mathrm{FB}}^{t}, p_{\mathrm{T}}^{t \bar{t}} \geq 20 \mathrm{GeV}$ & $-2.41 \pm 0.05$ & $-2.94 \pm 0.05$ & $-2.80 \pm 0.05$ & $-2.80 \pm 0.05$ & $-2.85 \pm 0.05$ & $-2.81 \pm 0.05$ \\
\hline$A_{\mathrm{FB}}^{t}, p_{\mathrm{T}}^{t \bar{t}} \geq 35 \mathrm{GeV}$ & $-3.90 \pm 0.06$ & $-3.85 \pm 0.05$ & $-3.54 \pm 0.06$ & $-3.55 \pm 0.06$ & $-3.67 \pm 0.06$ & $-3.63 \pm 0.06$ \\
\hline$A_{\mathrm{FB}}^{t}, p_{\mathrm{T}}^{t \bar{t}} \geq 50 \mathrm{GeV}$ & $-4.31 \pm 0.07$ & $-4.33 \pm 0.06$ & $-4.00 \pm 0.07$ & $-4.02 \pm 0.07$ & $-4.19 \pm 0.07$ & $-4.19 \pm 0.07$ \\
\hline$A_{\mathrm{FB}}^{t}, p_{\mathrm{T}}^{t \bar{t}} \geq 75 \mathrm{GeV}$ & $-4.88 \pm 0.08$ & $-4.62 \pm 0.08$ & $-4.33 \pm 0.09$ & $-4.29 \pm 0.09$ & $-4.59 \pm 0.09$ & $-4.56 \pm 0.09$ \\
\hline$A_{\mathrm{FB}}^{t \bar{t}}$ total & $-4.40 \pm 0.04$ & $-4.34 \pm 0.05$ & $-2.80 \pm 0.11$ & $-2.54 \pm 0.11$ & $-2.22 \pm 0.11$ & $-1.84 \pm 0.11$ \\
\hline$A_{\mathrm{FB}}^{t \bar{t}},\left|\Delta y_{t \bar{t}}\right|<1.0$ & $-2.70 \pm 0.04$ & $-2.62 \pm 0.05$ & $-1.71 \pm 0.11$ & $-1.91 \pm 0.11$ & $-1.39 \pm 0.11$ & $-1.16 \pm 0.11$ \\
\hline$A_{\mathrm{FB}}^{t \bar{t}},\left|\Delta y_{t \bar{t}}\right| \geq 1.0$ & $-19.48 \pm 0.18$ & $-19.54 \pm 0.22$ & $-10.52 \pm 0.52$ & $-9.75 \pm 0.51$ & $-9.22 \pm 0.52$ & $-7.54 \pm 0.51$ \\
\hline$A_{\mathrm{FB}}^{t \bar{t}},\left|m_{t \bar{t}}\right|<450 \mathrm{GeV}$ & $-3.59 \pm 0.06$ & $-3.51 \pm 0.06$ & $-2.67 \pm 0.14$ & $-2.36 \pm 0.13$ & $-1.74 \pm 0.14$ & $-1.63 \pm 0.14$ \\
\hline$A_{\mathrm{FB}}^{t \bar{t}},\left|m_{t \bar{t}}\right| \geq 450 \mathrm{GeV}$ & $-5.70 \pm 0.06$ & $-5.66 \pm 0.08$ & $-3.03 \pm 0.19$ & $-2.88 \pm 0.18$ & $-3.06 \pm 0.18$ & $-2.20 \pm 0.18$ \\
\hline$A_{\mathrm{FB}}^{t \bar{t}}, p_{\mathrm{T}}^{t \bar{t}} \geq 10 \mathrm{GeV}$ & $-4.35 \pm 0.04$ & $-4.32 \pm 0.05$ & $-3.98 \pm 0.06$ & $-3.86 \pm 0.06$ & $-3.72 \pm 0.06$ & $-3.51 \pm 0.06$ \\
\hline$A_{\mathrm{FB}}^{t \bar{t}}, p_{\mathrm{T}}^{t \bar{t}} \geq 20 \mathrm{GeV}$ & $-3.71 \pm 0.05$ & $-4.29 \pm 0.05$ & $-4.22 \pm 0.05$ & $-4.18 \pm 0.05$ & $-4.15 \pm 0.05$ & $-4.11 \pm 0.05$ \\
\hline$A_{\mathrm{FB}}^{t \bar{t}}, p_{\mathrm{T}}^{t \bar{t}} \geq 35 \mathrm{GeV}$ & $-5.72 \pm 0.06$ & $-5.52 \pm 0.05$ & $-5.16 \pm 0.06$ & $-5.17 \pm 0.06$ & $-5.21 \pm 0.06$ & $-5.21 \pm 0.06$ \\
\hline$A_{\mathrm{FB}}^{t \bar{t}}, p_{\mathrm{T}}^{t \bar{t}} \geq 50 \mathrm{GeV}$ & $-6.25 \pm 0.07$ & $-6.11 \pm 0.06$ & $-5.70 \pm 0.07$ & $-5.74 \pm 0.07$ & $-5.85 \pm 0.07$ & $-5.92 \pm 0.07$ \\
\hline$A_{\mathrm{FB}}^{t \bar{t}}, p_{\mathrm{T}}^{t \bar{t}} \geq 75 \mathrm{GeV}$ & $-6.62 \pm 0.08$ & $-6.45 \pm 0.08$ & $-5.99 \pm 0.09$ & $-5.94 \pm 0.09$ & $-6.27 \pm 0.09$ & $-6.25 \pm 0.09$ \\
\hline
\end{tabular}

Table 3. Results for the forward-backward asymmetries $A_{\mathrm{FB}}^{t}$ and $A_{\mathrm{FB}}^{t \bar{t}}$ at the Tevatron, for the $t \bar{t} j$ sample with various acceptance cuts. Jets are reconstructed by the inclusive $k_{\mathrm{T}}$-algorithm with $R=1$, above the $p_{\mathrm{T}}^{\text {jet }}>20 \mathrm{GeV}$ minimum jet cut.

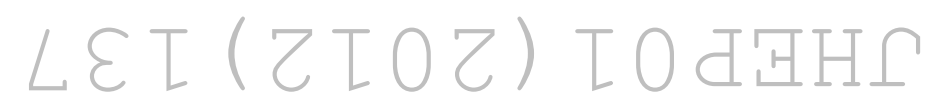


In table 3 we have also included our predictions for the forward-backward asymmetries with $p_{\mathrm{T}}^{t \bar{t}}>0$. We now see, that the results after showering with two different SMCs are consistent almost everywhere, apart from the very low $p_{\mathrm{T}}^{t \bar{t}}$ region, which thus contributes to generate the differences in $A_{\mathrm{FB}}^{t \bar{t}}$. Moreover, for both asymmetries $A_{\mathrm{FB}}^{t}$ and $A_{\mathrm{FB}}^{t \bar{t}}$, we observe that for $p_{\mathrm{T}}^{t \bar{t}}>10 \mathrm{GeV}$ the inclusion of the parton-shower leads to results in fair agreement with the NLO ones. The large differences between NLO and showered results observed in the total asymmetries are thus traced back to the region of small $p_{\mathrm{T}}^{t \bar{t}}$ s. This appears plausible since in that region soft and collinear emission produced by the parton shower will be more important compared to the large $p_{\mathrm{T}}^{t \bar{t}}$ region. We also note that our findings are different from the observations made in [48] where a stronger effect of the parton shower using specific tunes was observed over the entire $p_{\mathrm{T}}^{t \bar{t}}$ range. One has to remind, however, that for a leading-order SMC such as the PYTHIA program used in [48], the contributions to the asymmetry for $p_{\mathrm{T}}^{t \bar{t}}>0$ are generated exclusively by the shower. It thus looks feasible that a change in the parton shower will be reflected in a overall change of the predictions.

Whether the aforementioned large corrections produced by the parton shower could be responsible in part for the observed discrepancy between theory and experiment is however unclear because in the $t \bar{t}$ sample this effect may cancel. Given the stability of the theoretical prediction above $p_{\mathrm{T}}^{t \bar{t}}=10 \mathrm{GeV}$ —now available at NLO accuracy - a detailed comparison of experimental data and theory predictions may provide useful information to further scrutinize the observed discrepancy in the inclusive sample.

\section{$3.2 \quad \mathrm{LHC}$}

In figures 6 to 11 we report a similar set of distributions as already shown for the Tevatron, this time for the LHC collider at $\sqrt{s}=7 \mathrm{TeV}$. For most of the observables, the same comments and conclusions as reported for the Tevatron case are, in general, valid here, too. For this reason, we will not repeat the same remarks here. There are, however, some new plots that deserve discussion and explanations.

In the lower panel of figure 6 we show the differential cross section as a function of the rapidity distribution of the top-quark $y_{t}$. This inclusive quantity shows the expected good agreement between NLO and LHEF results. It is also pretty stable with respect to the inclusion of parton shower effects, resulting only in a few percent change in the overall normalization.

In the upper panel of figure 9 we show the differential cross section $d \sigma / d p_{\mathrm{T} \text {,rel }}^{j_{\text {st }}}$ as function of the scalar sum of the relative transverse momenta of the particles in the first jet, $p_{\mathrm{T}, \mathrm{rel}}^{j_{1 \mathrm{st}}}$, which is defined with respect to the jet axis in the frame where the jet has zero rapidity. In general, we define for the $n$-th jet,

$$
p_{\mathrm{T}, \mathrm{rel}}^{j_{n \mathrm{~h}}}=\sum_{i \in j_{n \text {th }}} \frac{\left|\vec{k}_{i} \times \vec{p}^{j_{n \mathrm{th}}}\right|}{\left|\vec{p}^{j_{n \mathrm{th}}}\right|},
$$

where $k_{i}$ denotes the momentum of the $i^{\text {th }}$ particle in the $n$-th jet, see also [50]. The observable behaves as expected: The NLO curve shows the unphysical enhancement associated with the IR singularity at $p_{\mathrm{T}, \text { rel }}^{j_{1 \mathrm{~s}}}=0$, the LHEF result corrects for this only partially, owing to the Sudakov suppression by final-state radiation, while the fully showered events 

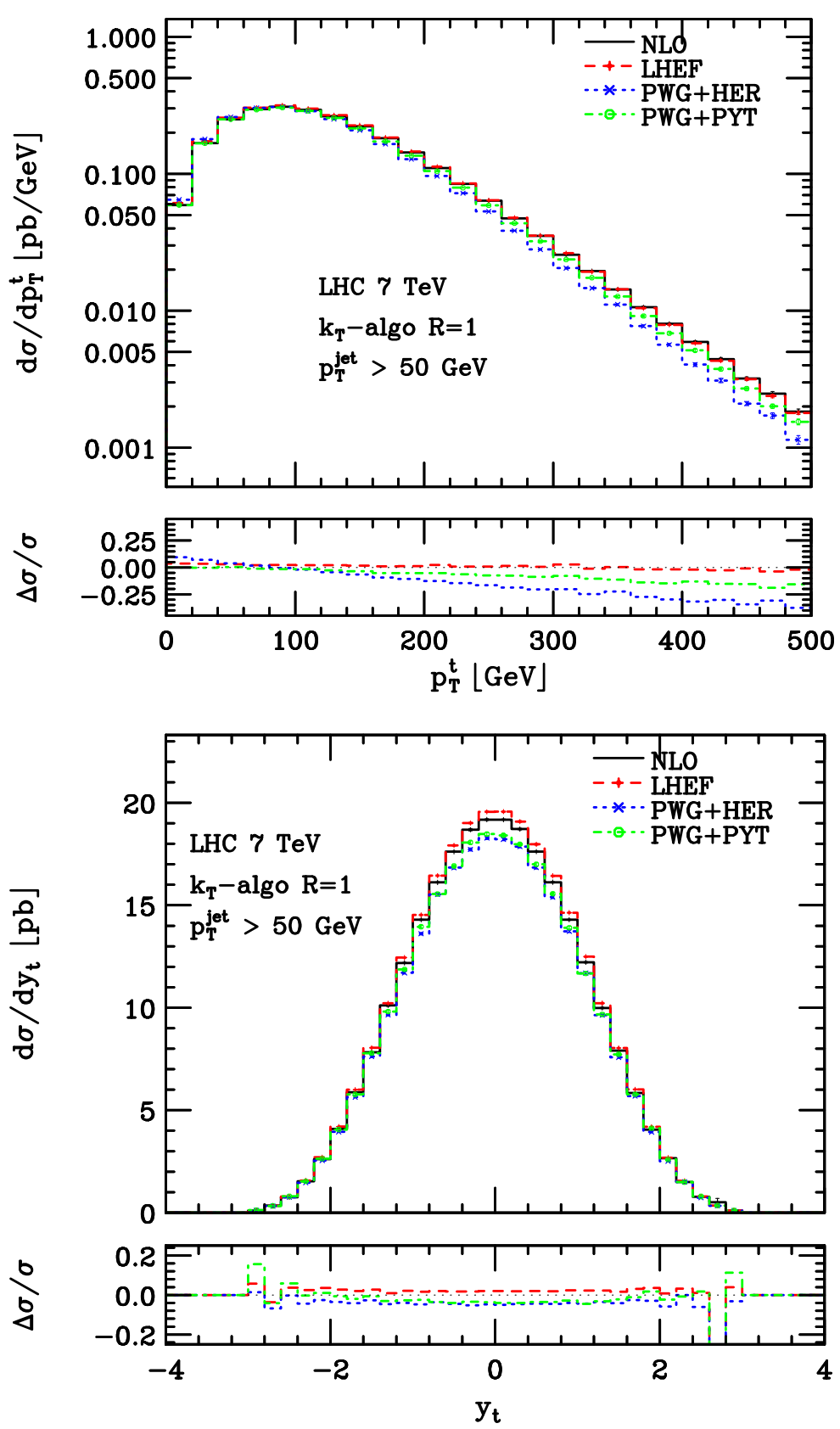

Figure 6. The differential cross sections as function of the transverse momentum $p_{\mathrm{T}}^{t}$ and of the rapidity $y_{t}$ of the top-quark at the $\mathrm{LHC}(\sqrt{s}=7 \mathrm{TeV})$.

manifest the physical suppression at low $p_{\mathrm{T}, \text { rel }}^{j_{1 \text { st }}}$. Moreover, in the lower panel of figure 9 we show the distribution of the invariant mass of the system made by the top-quark and the hardest light jet.

Eventually, in the lower panel of figure 10, the total exclusive cross section as a function of the number of resolved jets, $N_{\text {jets }}$, is shown. The moderate difference in the $N_{\text {jets }}=2$ bin between the NLO and LHEF results may be considered a consequence of the differ- 

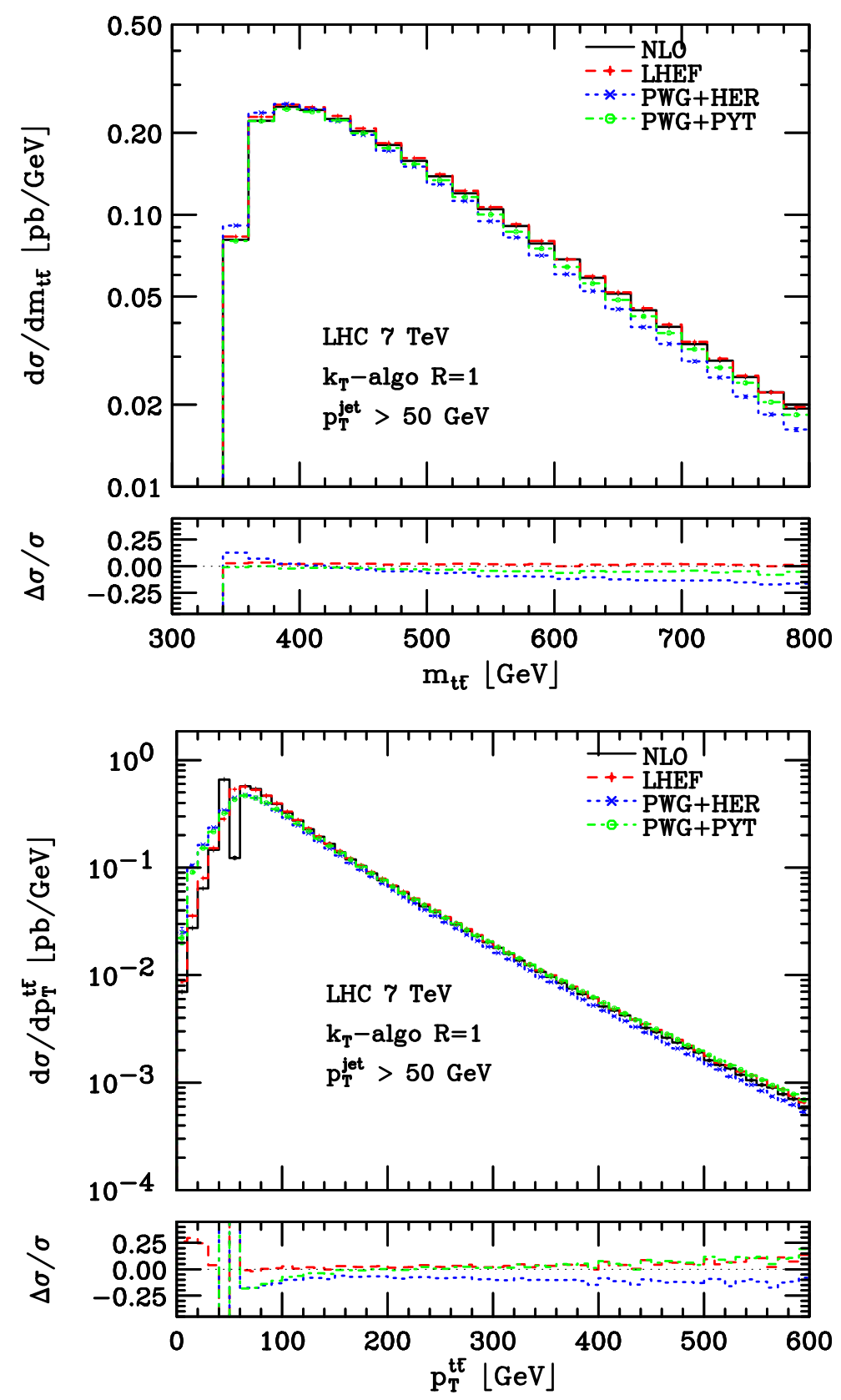

Figure 7. The differential cross sections as function of the $t \bar{t}$-pair invariant mass $m_{t \bar{t}}$ and transverse momentum $p_{\mathrm{T}}^{t \bar{t}}$ at the $\operatorname{LHC}(\sqrt{s}=7 \mathrm{TeV})$.

ent normalization of the distributions involving the next-to-hardest jet, as shown e.g., in figure 11. Higher bins are instead populated only by the shower and thus predictions for those bins are only accurate in the strict soft/collinear limit.

One noticeable difference w.r.t. Tevatron results is the reduced discrepancy between the showered and partonic results for observables that involve the hardest or next-tohardest jets, e.g., figures 8 and 11. A possible explanation for this is in the higher jet 

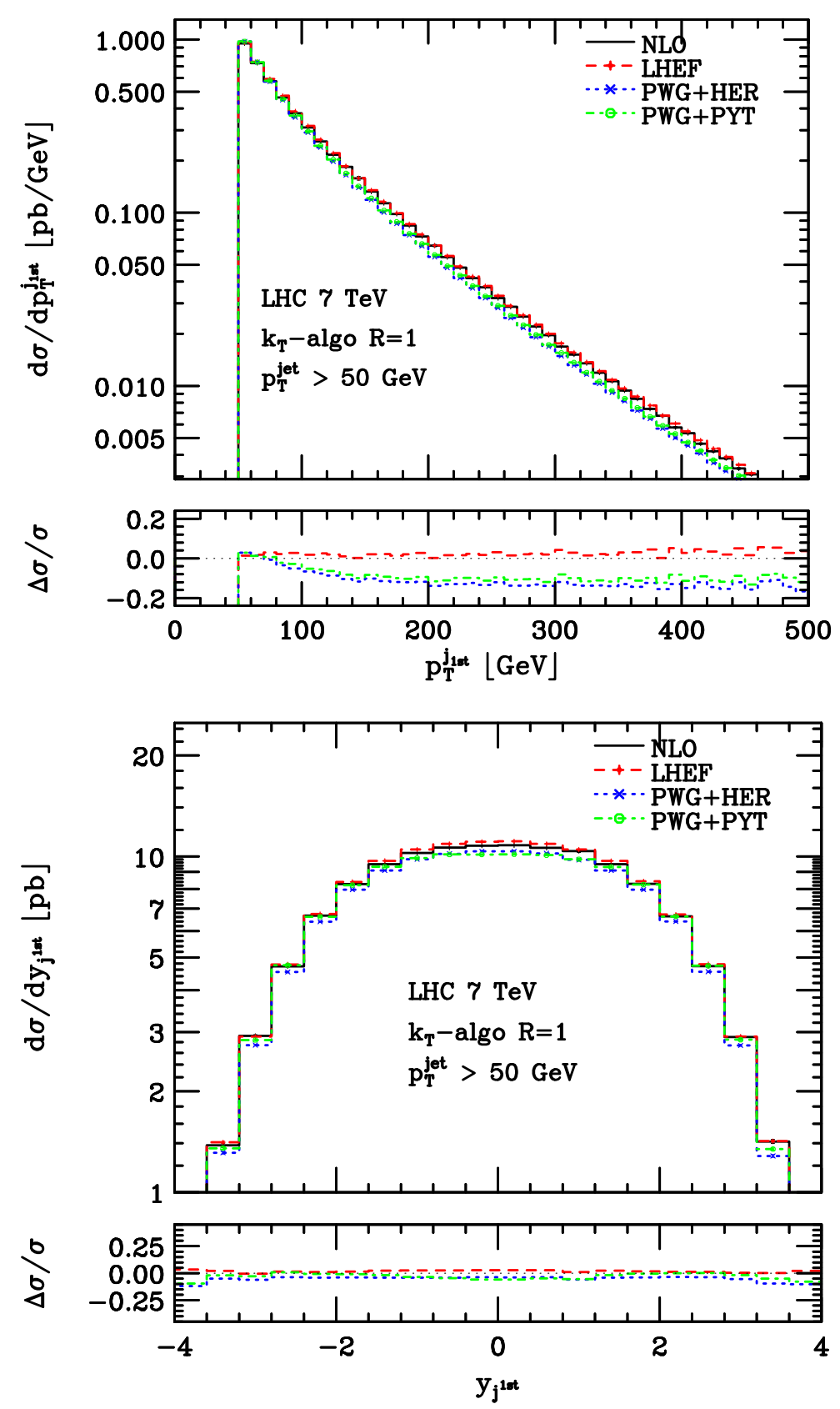

Figure 8. The differential cross sections as function of the hardest jet transverse momentum and rapidity at the $\operatorname{LHC}(\sqrt{s}=7 \mathrm{TeV})$.

cut $\left(p_{\mathrm{T}}=50 \mathrm{GeV}\right)$ used for LHC analysis, that largely restricts the regions where shower effects may become important.

A first phenomenological application of the POWHEG implementation discussed here has already been reported in [54], namely the study of $t \bar{t} j$-events at the LHC as a function of the invariant mass of the multi-jet systems as a means of measuring the top-quark mass. 

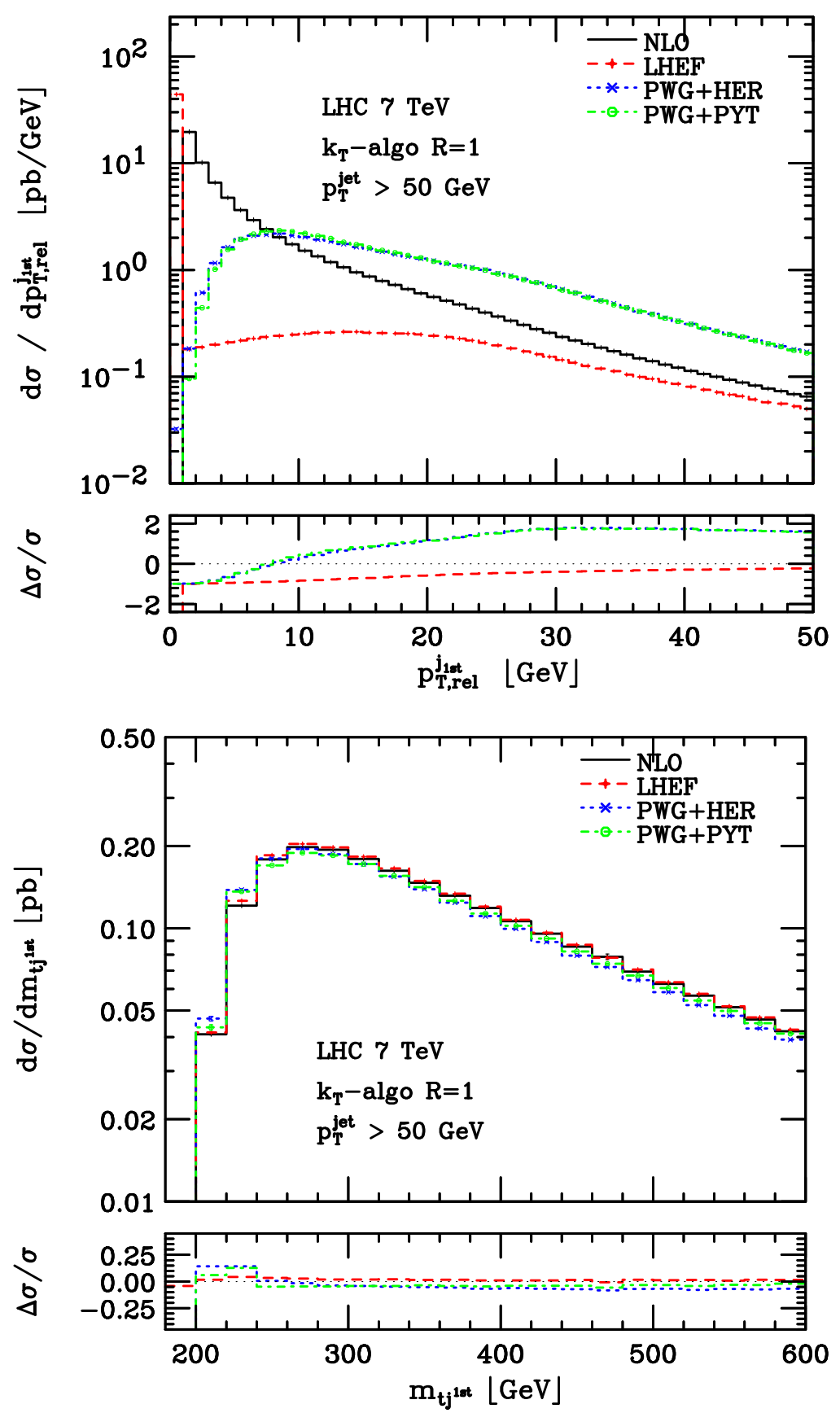

Figure 9. The differential cross sections as function of the scalar sum of transverse momenta inside the hardest jet (upper panel), as defined in eq. (3.2), and the invariant mass of the system made by the top-quark and the hardest jet (lower panel), at the LHC $(\sqrt{s}=7 \mathrm{TeV})$.

\subsubsection{Top-quark charge asymmetry}

In table 4 we present our estimate of the charge asymmetries in the $t \bar{t} j$ sample, measured with respect to the (anti-) top-quark pseudo-rapidity $\eta$ and rapidity $y$, at various stages of 

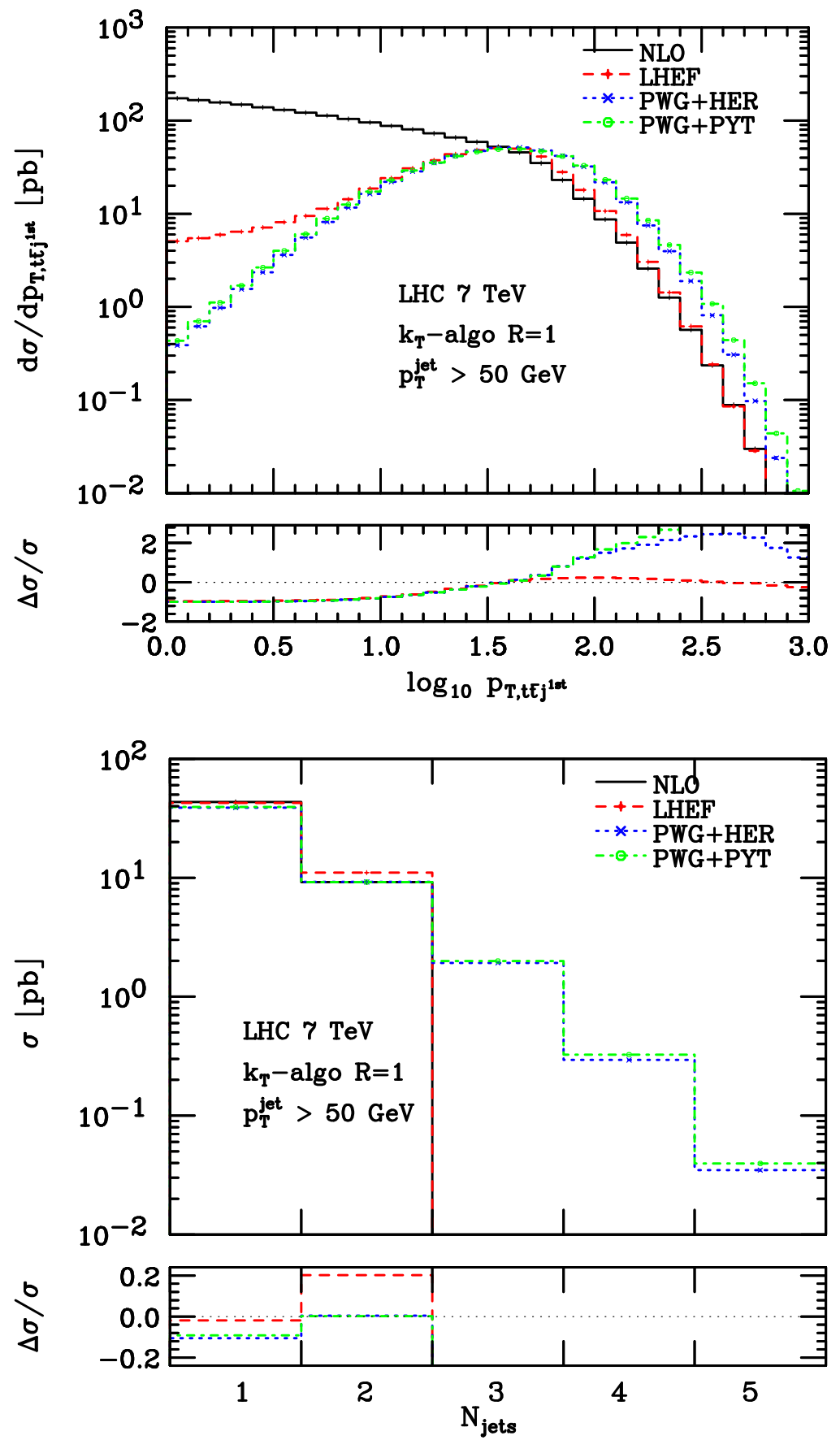

Figure 10. The differential cross sections as function of the transverse momentum of the system made by $t \bar{t}$-pair and the hardest jet (upper panel) and the total inclusive cross section for $\sigma_{\geq N_{\text {jets }}}$ (lower panel) at the $\mathrm{LHC}(\sqrt{s}=7 \mathrm{TeV})$.

our simulation. The two observables are defined in the same way

$$
A_{C}^{x}=\frac{1}{\sigma}\left(\int_{\Delta|x|>0} d \sigma-\int_{\Delta|x|<0} d \sigma\right),
$$



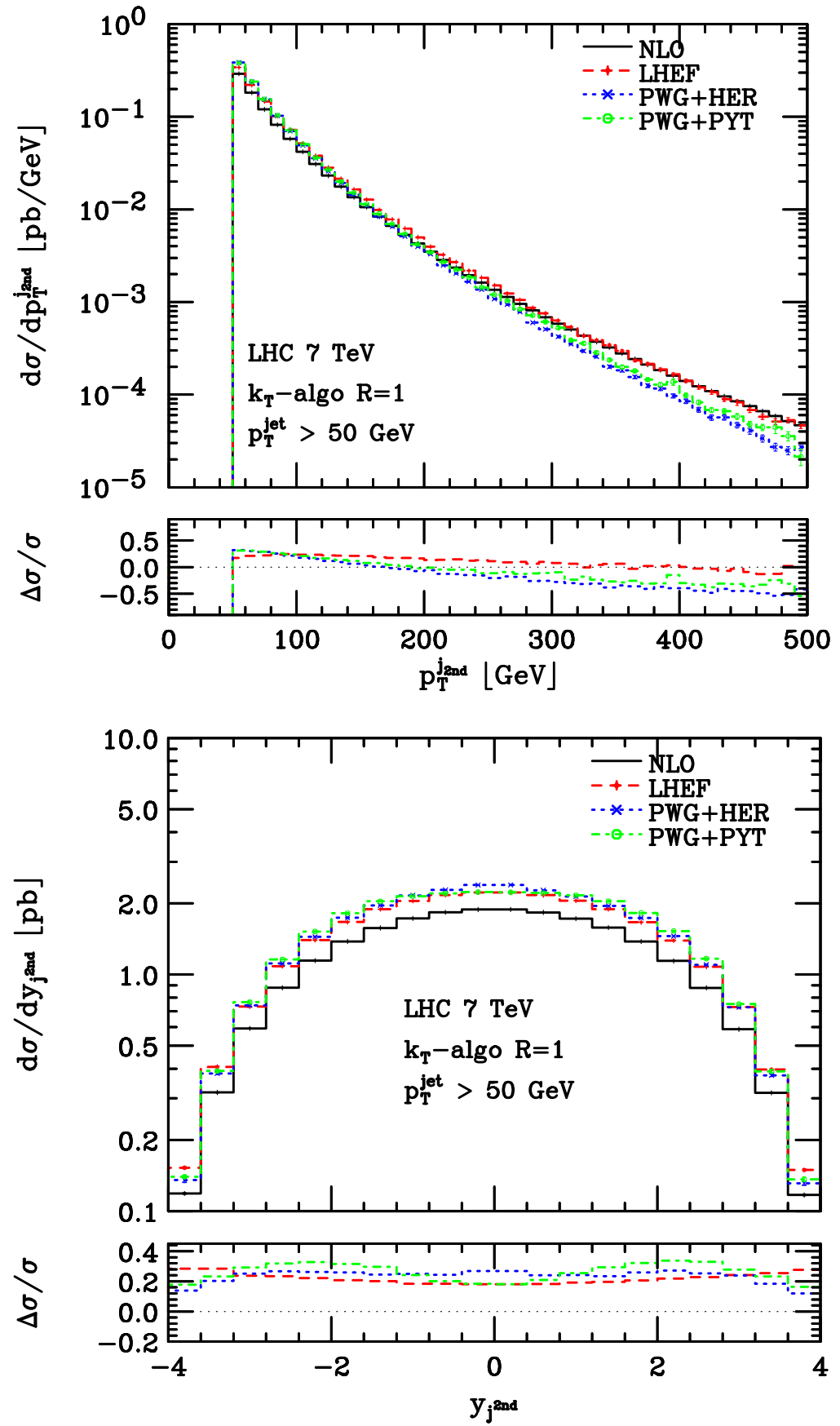

Figure 11. The differential cross sections as function of the next-to-hardest jet transverse momentum and rapidity at the $\operatorname{LHC}(\sqrt{s}=7 \mathrm{TeV})$.

with $\Delta|x|=\left|x_{t}\right|-\left|x_{\bar{t}}\right|$ and $x=\eta$ or $x=y$, respectively. By looking at our results, one can see that a positive asymmetry is present for both observables, albeit larger in magnitude for $A_{C}^{y}$. Moreover, results obtained with the inclusion of PYTHIA and HERWIG showers are in fair agreement, even after the addition of MPI or UE. We also observe that the numerical evaluation of the asymmetry is rather challenging at fixed order, leading to large 


\begin{tabular}{|ccccccc|}
\hline LHC $7 \mathrm{TeV}$ & $\begin{array}{c}\text { NLO } \\
{[\%]}\end{array}$ & $\begin{array}{c}\text { LHEF } \\
{[\%]}\end{array}$ & $\begin{array}{c}\text { PWG+HER } \\
{[\%]}\end{array}$ & $\begin{array}{c}\text { PWG+HER+UE } \\
{[\%]}\end{array}$ & $\begin{array}{c}\text { PWG+PYT } \\
{[\%]}\end{array}$ & $\begin{array}{c}\text { PWG+PYT+MPI } \\
{[\%]}\end{array}$ \\
\hline$A_{C}^{\eta}$ & $0.19 \pm 0.09$ & $0.18 \pm 0.06$ & $0.46 \pm 0.10$ & $0.26 \pm 0.11$ & $0.40 \pm 0.11$ & $0.57 \pm 0.11$ \\
$A_{C}^{y}$ & $0.51 \pm 0.09$ & $0.47 \pm 0.06$ & $0.73 \pm 0.10$ & $0.52 \pm 0.11$ & $0.66 \pm 0.11$ & $0.76+0.11$ \\
\hline
\end{tabular}

Table 4. Results for the top-quark charge asymmetry $A_{C}$ in the $t \bar{t} j$ sample at the LHC $(\sqrt{s}=$ $7 \mathrm{TeV})$. Jets are reconstructed by the inclusive $k_{\mathrm{T}}$-algorithm with $R=1$, above the $p_{\mathrm{T}}^{\text {jet }}>50 \mathrm{GeV}$ minimum jet cut.

\begin{tabular}{|cccccc|}
\hline Tevatron 1.96 TeV & LHEF & PWG+HER & PWG+HER+UE & PWG+PYT & PWG+PYT+MPI \\
& {$[\%]$} & {$[\%]$} & {$[\%]$} & {$[\%]$} & {$[\%]$} \\
\hline$A_{\mathrm{FB}}^{\ell^{+}}$ & $-1.83 \pm 0.05$ & $-0.94 \pm 0.11$ & $-0.92 \pm 0.11$ & $-0.57 \pm 0.11$ & $-0.41 \pm 0.11$ \\
$A_{\mathrm{FB}}^{\ell^{+} \ell^{-}}$ & $-2.21 \pm 0.05$ & $-1.00 \pm 0.11$ & $-0.99 \pm 0.11$ & $-0.59 \pm 0.11$ & $-0.46 \pm 0.11$ \\
\hline & PWG+HER' & PWG+HER'+UE & PWG+PYT' & PWG+PYT'+MPI \\
& {$[\%]$} & {$[\%]$} & {$[\%]$} & {$[\%]$} \\
\hline$A_{\mathrm{FB}}^{\ell^{+}}$ & & $-0.69 \pm 0.11$ & $-0.93 \pm 0.11$ & $-0.71 \pm 0.11$ & $-0.47 \pm 0.11$ \\
$A_{\mathrm{FB}}^{\ell^{+} \ell^{-}}$ & & $-1.13 \pm 0.11$ & $-1.11 \pm 0.11$ & $-0.90 \pm 0.11$ & $-0.85 \pm 0.11$ \\
\hline
\end{tabular}

Table 5. Results for the lepton charge asymmetries $A_{\mathrm{FB}}^{\ell^{+}}$and $A_{\mathrm{FB}}^{\ell^{+} \ell^{-}}$in the $t \bar{t} j$ sample at the Tevatron $(\sqrt{s}=1.96 \mathrm{TeV})$. Jets are reconstructed by the inclusive $k_{\mathrm{T}}$-algorithm with $R=1$, above the $p_{\mathrm{T}}^{\text {jet }}>20 \mathrm{GeV}$ minimum jet cut.

statistical uncertainties. Only after increasing the statistics by one order of magnitude we were able to obtain errors comparable with those obtained after the inclusion of the parton shower. We consider the results including the effects of the parton shower as the best prediction currently available for the asymmetry. We refer to refs. $[55,56]$ for recent LHC measurements of $A_{C}^{y}$ and $A_{C}^{\eta}$ in the $t \bar{t}$ inclusive sample.

In closing we remark, that we have also carefully tested the LHC nominal center-ofmass energy, producing the same set of distributions as in figures 6 to 11 for $\sqrt{s}=14 \mathrm{TeV}$ collisions. Since no significant difference in the behaviour has been observed in any of the distributions, with respect to the $7 \mathrm{TeV}$ case, we refrain from including the corresponding plots here.

\subsection{Top-quark decay}

The studies so far have been based on the assumption of stable (anti-)top-quarks during both the fixed order calculation stage and the generation of the hardest radiation according to the POWHEG method. Thus, the output resulting from these stages includes on-shell topquarks and extra light partons only. When interfacing to a SMC program for performing the rest of the showering and the hadronization steps, no information concerning the spin or helicity of these top-quarks is retained and the decay is performed by the SMC, which averages over the possible top polarizations.

In this way one does not correctly account for top-quark off-shell effects, non-resonant production mechanisms and for the top-quark spin correlations. However, given the large ratio between the top-quark mass and the top-quark width, the first two effects are suppressed as $\mathcal{O}\left(\Gamma_{t} / m_{t}\right)$ and, therefore, are rather modest. This was also explicitly shown for 
the $t \bar{t}$ production case by the recent calculation of the NLO corrections to the $W^{+} W^{-} b \bar{b}$ final state $[57,58]$.

Top-quark spin correlations at hadron colliders were instead realized to be an important tool to study top-quark properties since the early work of [59]. Also, the QCD corrected spin analyzing power of jets in decays of polarized top-quarks has been extensively discussed in [60]. The possibility to measure these correlation effects is particular to top-quarks because of their large mass and short lifetime, which prevent non-perturbative QCD effects from significantly depolarizing them before the decay.

The usual approach to study this phenomenon relies on taking the top-quark zero-width limit and separately considering NLO corrections to the production and decay processes. This is done by means of a spin correlation matrix which accounts for all the possible spin configurations occurring in the two stages. Several studies following this approach have already been presented, concerning both $t \bar{t}$ production [59] with spin correlations at NLO and the $t \bar{t} j$ case $[20]$ with spin correlations accounted for at leading order.

Here we follow a different approach, that was first introduced in [61] and has already been employed and discussed in both implementations, POWHEG [18, 19, 62] and MC@NLO $[16,63]$. In this way we avoid having to introduce the spin correlation matrices, but we nevertheless recover the exact leading-order spin correlations. To be more precise, we have LO spin-correlations in the soft and collinear regions, while the same accuracy of the complete NLO calculation, namely the matrix element,

$$
p p(p \bar{p}) \rightarrow\left(t \rightarrow b \ell^{+} \nu\right)\left(\bar{t} \rightarrow \bar{b} \ell^{-} \bar{\nu}\right) j j,
$$

is used for the hard regions. ${ }^{5}$ For the sake of brevity, we explain this method here only briefly, and instead, refer to the aforementioned publications for more details. The basic idea is to first generate events with stable top-quarks (un-decayed events) through the usual POWHEG machinery and then generate the decay products according to the matrix element for the full production and decay process (decayed events).

Moreover, in order to produce more realistic final states, we have also allowed for a reshuffling of the momenta of the top-quark decay products, resulting in off-shell topquarks and $W$ bosons, whose virtualities have been distributed according to Breit-Wigner shapes. This reshuffling is such that the relative 3-momentum of the (anti-)top-quark in the $t \bar{t}$ rest frame and the 3-momentum of the radiated light partons in the partonic centerof-mass system are kept fixed. It also takes care of the small changes in the phase space and luminosity due to the $t$ and $\bar{t}$ quark being slightly off-shell with respect to the chosen pole mass value. In any case, we restrict the top-quark and $W$ virtualities off-shellness to be in between 10 widths. We have checked that this modification does not significantly change the results obtained in the top-quark and $W$ boson zero-width approximation, by comparing several distributions for the two cases. ${ }^{6}$

\footnotetext{
${ }^{5} \mathrm{In}$ case no radiation harder than $p_{\mathrm{T}}^{\min }=0.8 \mathrm{GeV}$ is generated by the POWHEG method, the event is classified as Born-like: the corresponding matrix elements for the decayed process, i.e., $p p(p \bar{p}) \rightarrow(t \rightarrow$ $\left.b \ell^{+} \nu\right)\left(\bar{t} \rightarrow \bar{b} \ell^{-} \bar{\nu}\right) j$, are then employed.

${ }^{6}$ The zero-width approximation in the decay may always be enforced by setting the zerowidth token to 1 in the input card.
} 
Furthermore, since charged leptons (or down-type quarks) coming from the top-quark decays are the most sensitive probe of the top-quark spin directions, we concentrate on the di-leptons channel only. We thus always assume $t \rightarrow W^{+} b \rightarrow \ell^{+} \nu b$ and $\bar{t} \rightarrow W^{-} \bar{b} \rightarrow \ell^{-} \bar{\nu}_{\ell} \bar{b}$, with branching ratio $B R(W \rightarrow \ell \nu)=0.108$. However, we stress that our implementation is completely general for what concerns the top-quark decay products, such that the lepton+jets or the all hadronic search channels can be simulated as well, if desired. ${ }^{7}$

Thus, taking as an example the semi-leptonic top-quark decay channel, the conditional probability for the decayed events, starting from un-decayed ones, is obtained from

$$
d P\left(\Phi_{\text {dec. }} \mid \Phi_{\text {undec. }}\right)=\frac{1}{\operatorname{BR}(t \rightarrow b \bar{\ell} \nu) \operatorname{BR}(\bar{t} \rightarrow \bar{b} \ell \bar{\nu})} \frac{\mathcal{M}_{\text {dec. }}\left(\Phi_{\text {undec. }}, \Phi_{t \rightarrow b \bar{\ell} \nu}, \Phi_{\bar{t} \rightarrow \bar{b} \ell \bar{\nu}}\right)}{\mathcal{M}_{\text {undec. }}\left(\Phi_{\text {undec. }}\right)} d \Phi_{t \rightarrow b \bar{\ell} \nu} d \Phi_{\bar{t} \rightarrow \bar{b} \ell \bar{\nu}},
$$

where $\Phi_{\text {dec. }}=\left\{\Phi_{\text {undec. }}, \Phi_{t \rightarrow b \bar{\ell} \nu}, \Phi_{\bar{t} \rightarrow \bar{b} \ell \bar{\nu}}\right\}$ is the full space phase including the decay products, while $\mathcal{M}$ 's are the matrix elements squared for the given partonic process. In practice, however, to generate $\Phi_{\text {dec. }}$ configurations according to eq. (3.5), one makes use of an upper bound $U_{\text {dec. }}$ for the ratio $\mathcal{M}_{\text {dec. }} / \mathcal{M}_{\text {undec. }}$ and then proceed as follows:

I. Generate a tentative decay kinematics $\Phi_{\text {dec. }}$, starting from $\Phi_{\text {undec. }}$.

II. Extract a random value $r$ in the range [0, $\left.U_{\text {dec. }}\right]$.

III. If $r<\mathcal{M}_{\text {dec. }}\left(\Phi_{\text {dec. }}\right) / \mathcal{M}_{\text {undec. }}\left(\Phi_{\text {undec. }}\right)$, then the decay kinematics is allowed and the event is accepted. Otherwise go back to step I.

An efficient functional form of the upper-bounding function may be readily reconstructed from the structure of top-quarks decay:

$$
U_{\text {dec. }}\left(\Phi_{\text {dec. }}\right)=\mathcal{N} \frac{\mathcal{M}_{t \rightarrow b W}\left(M_{t}^{2}, M_{\bar{\ell} \nu}^{2}\right)}{\left(M_{t}^{2}-m_{t}^{2}\right)^{2}+m_{t}^{2} \Gamma_{t}^{2}} \frac{\mathcal{M}_{W \rightarrow \bar{\ell} \nu}\left(M_{\bar{\ell} \nu}^{2}\right)}{\left(M_{\bar{\ell} \nu}^{2}-m_{W}^{2}\right)^{2}+m_{W}^{2} \Gamma_{W}^{2}} \times(t \leftrightarrow \bar{t}),
$$

where $M_{t}^{2}$ and $M_{\bar{\ell} \nu}^{2}=\left(k_{\bar{\ell}}+k_{\nu}\right)^{2}$ are the top-quark and $\mathrm{W}$ boson virtualities and $\mathcal{M}_{t \rightarrow b W}$ and $\mathcal{M}_{W \rightarrow \bar{\ell} \nu}$ are the squared amplitudes for the $1 \rightarrow 2$ decay processes. The choice of the normalization factor $\mathcal{N}$ has been performed by sampling the allowed phase space, by requiring that the inequality

$$
\mathcal{M}_{\text {dec. }}^{f}\left(\Phi_{\text {undec. }}, \Phi_{\text {dec. }}\right) \leq \mathcal{M}_{\text {undec. }}^{f}\left(\Phi_{\text {undec. }}\right) U_{\text {dec. }}\left(\Phi_{\text {dec. }}\right)
$$

always holds, for any given specific subprocess flavour $f$.

In the rest of this section we present results obtained within this approach. We have set

$$
\begin{gathered}
\Gamma_{t}=1.31 \mathrm{GeV}, \quad \mathrm{m}_{\mathrm{W}}=80.398 \mathrm{GeV}, \quad \Gamma_{\mathrm{W}}=2.141 \mathrm{GeV}, \\
\alpha_{\mathrm{EM}}=1 / 128.89, \quad \sin ^{2} \theta_{W}=0.22265,
\end{gathered}
$$

and we have considered a simplified form for the CKM matrix, with a mixing between the first two generations only:

$$
V_{u d}=V_{c s}=0.975, \quad V_{u s}=V_{c d}=0.222, \quad V_{t b}=1
$$

\footnotetext{
${ }^{7}$ See e.g., [64] for a review of observables sensitive to spin-correlation effects in the lepton+jets channel.
} 
and all the other entries set to zero. No extra acceptance cuts on the leptons are placed in the following plots.

With these ingredients we are able to offer predictions for top-quarks decay products, at the LHEF level and after shower and hadronization. We compare them in figure 12 , where we plot the positively charged lepton transverse momentum and the rapidity of the negatively charged one, at the $7 \mathrm{TeV}$ LHC and $1.96 \mathrm{TeV}$ Tevatron colliders, respectively. We note that in these distributions shower effects are rather small. Moreover, one does not expect to see any dependence on the top-quark spin.

Spin correlation effects in $t \bar{t}$ production are usually studied by choosing $t$ and $\bar{t}$ spin quantization axis and looking at reference frames and angular distributions that are particularly sensitive to these correlations, ${ }^{8}$ a procedure that can easily be performed in SMC simulations. However, due to the presence of unobserved neutrinos, this can become a difficult task in the data analysis for an experimental collaboration. An example of such observables is the double differential distribution,

$$
\frac{1}{\sigma} \frac{d^{2} \sigma}{d \cos \theta_{1} d \cos \theta_{2}}
$$

where $\theta_{1}$ and $\theta_{2}$ are the angles between the directions of the flights of the leptons coming from the decayed top-quark in the $t(\bar{t})$ rest frame and an arbitrary direction that defines the quantization axis for the (anti-)top-quark spin.

The double differential distribution in eq. (3.10) can be evaluated to good approximation to

$$
\frac{1}{4}\left(1-\kappa \cos \theta_{1} \cos \theta_{2}\right)
$$

which is only valid, though, for fully inclusive cross sections. As soon as cuts are imposed the form of the distribution will change and a further dependence on $\cos \theta_{1,2}$ could be introduced. ${ }^{9}$ The cuts may also affect the simple interpretation of $\kappa$, which in general depends also on the choice of the spin quantization axis. Nevertheless even in the presence of cuts the distribution is still a useful observable to study the impact of spin correlations. This quantity has been recently measured by D0 [66] for the choice of the beam direction as quantization axis, finding $\kappa=0.10 \pm 0.45$. Due to the large uncertainties, this value is both compatible with no spin correlation and with the NLO predictions $\kappa=0.777$ of [59].

In figure 13 we present our predictions for the distribution in eq. (3.10) at the Tevatron using the beam-axis to define the lepton angles, obtained by including the spin correlation effects as explained above in our $t \bar{t} j$ sample. We compare these results with those obtained by letting the SMC program performing the uncorrelated top-quark decays. The effect of the spin correlations is clearly visible, leading to differences as large as $50 \%$ at extreme $\cos \theta_{1} \cos \theta_{2}$ values.

\footnotetext{
${ }^{8}$ See e.g., [65] for strategies to maximize the spin correlation of $t \bar{t}$-pairs produced at the LHC.

${ }^{9}$ We note that electroweak effects and QCD absorptive parts lead to a tiny polarization of the top-quark and thus a deviation from the simple form in eq. (3.11) of the double differential distribution shown in eq. (3.10), even in the absence of cuts.
} 

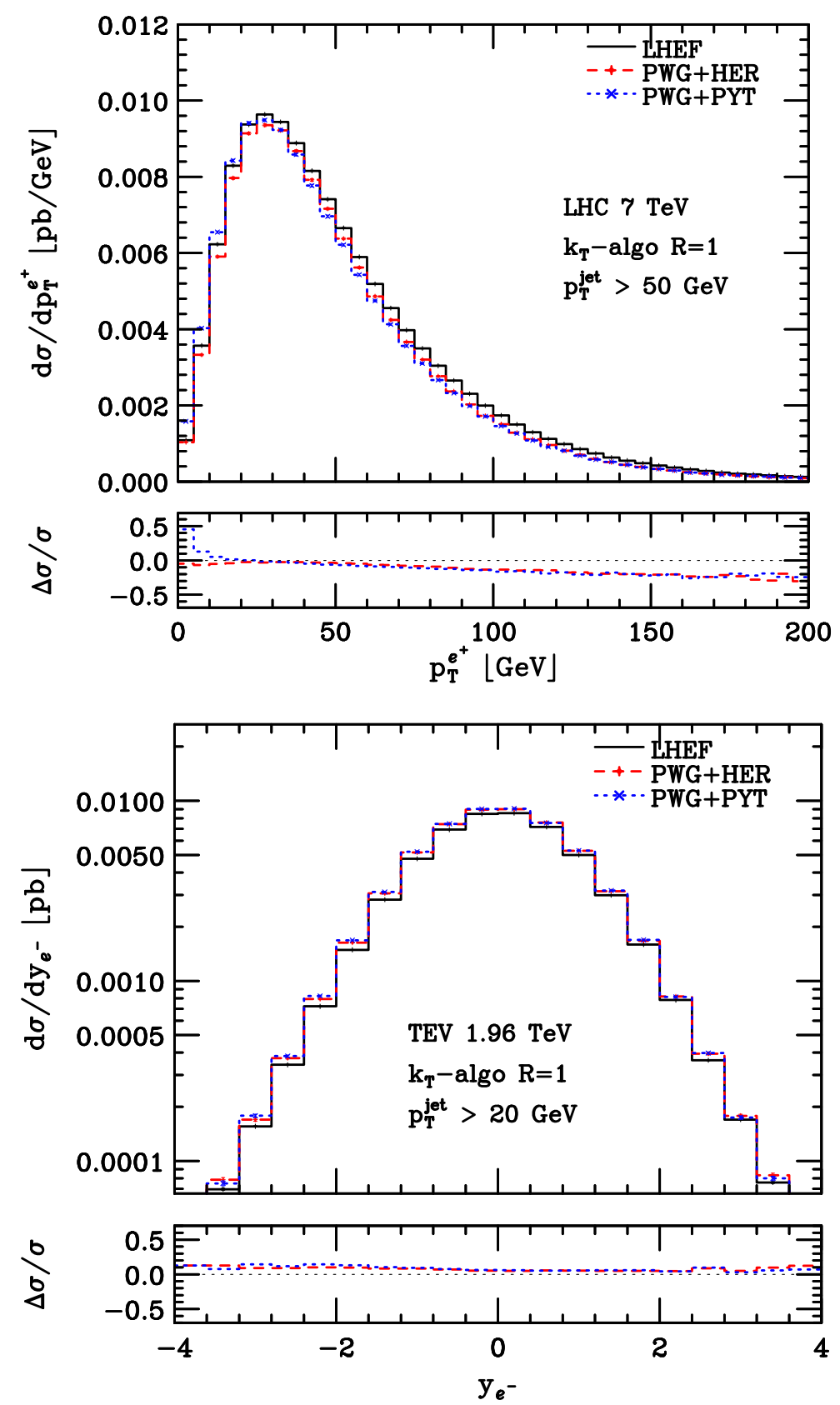

Figure 12. The differential cross sections as function of the positively charged lepton transverse momenta at the LHC $(\sqrt{s}=7 \mathrm{TeV})$ and the the differential cross sections as function of the negatively charged lepton rapidity at the Tevatron $(\sqrt{s}=1.96 \mathrm{TeV})$.

Alternatively, one can look at the distributions of the top-quark decay products in the laboratory frame. In this case the problem is to devise clever enough cuts to single out spin-correlation effects which are usually hardly visible. ${ }^{10}$

\footnotetext{
${ }^{10} \mathrm{~A}$ recent paper [67] suggests the use of these distributions to construct a likelihood function for the spincorrelation hypothesis, that can be then tested statistically for its validity. See also [68, 69] for up-to-date results using this approach at Tevatron.
} 

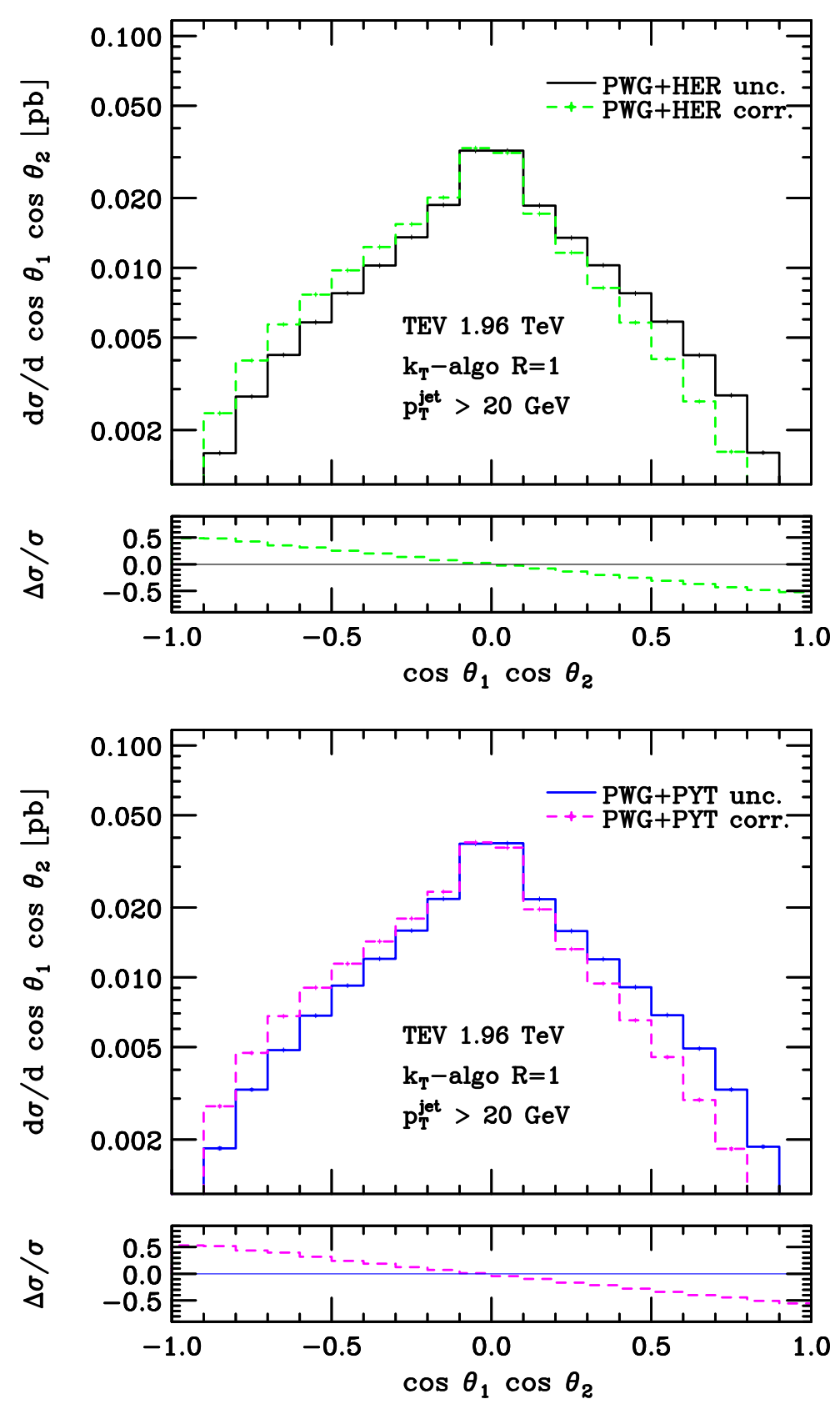

Figure 13. Effect of the inclusion of spin correlations when interfacing to HERWIG (upper) and PYTHIA (lower) programs. Results for the Tevatron collider $(\sqrt{s}=1.96 \mathrm{TeV})$.

There are, however, notable exceptions. One such example, the azimuthal distance between the leptons coming from the top- and the anti-top-quark, has already been shown in [70] to depend mildly on spin-correlations. Results for this observable has also been presented in [71], for the ongoing $\mathrm{LHC} 7 \mathrm{TeV}$ run. Later on, it has been suggested that imposing the $m_{t \bar{t}}<400 \mathrm{GeV}$ acceptance cut should enhance this dependence in the dilepton search channel at the LHC [64]. However, due to the inherent ambiguities of the 

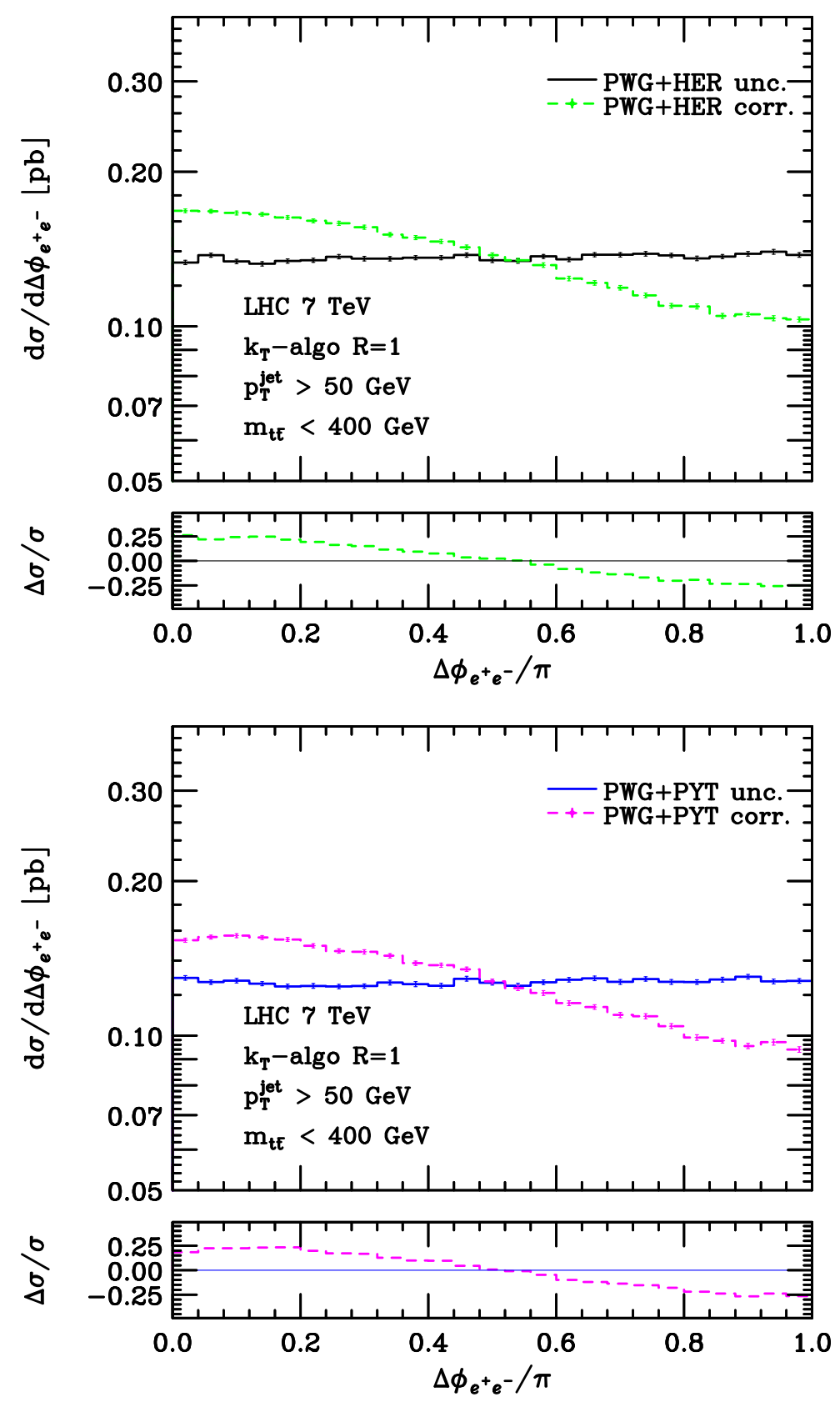

Figure 14. Effect of the inclusion of spin correlations when interfacing to HERWIG (upper) and PYTHIA (lower) programs. Results for the LHC collider $(\sqrt{s}=7 \mathrm{TeV})$.

unobserved neutrinos, the true $m_{t \bar{t}}$ value cannot be reconstructed on an event-by-event basis in a real experiment. In our case, we simply looked back in the shower history to reconstruct unambiguously the true $m_{t \bar{t}}$. A more realistic study would use the definition of $m_{t \bar{t}}$ value averaged over all the possible neutrino assignments proposed by the authors of [64]. However, as shown in [64], this only leads to minor differences.

In figure 14 we show our results with both the HERWIG and PYTHIA showers and the 
hadronization stage simulations. In both panels, spin correlation effects are again clearly visible as a change in the slope of the distribution.

As a final remark, we investigate the effects induced by top-quark charge asymmetries on the di-lepton final state, by studying the leptonic charge asymmetries. This issue has also been addressed in a recent paper [72], for the $t \bar{t}$ inclusive production, including nextto-leading order QCD and EW corrections. In ref. [20], the authors provide predictions for this quantity at NLO in the $t \bar{t} j$ sample. The definitions for the asymmetries follow the ones in eq. (3.1), but are expressed in term of leptonic rapidities in the laboratory frame:

$$
A_{\mathrm{FB}}^{\ell^{+}}=\frac{1}{\sigma}\left(\int_{y_{\ell^{+}}>0} d \sigma-\int_{y_{\ell^{+}}<0} d \sigma\right), \quad A_{\mathrm{FB}}^{\ell^{+} \ell^{-}}=\frac{1}{\sigma}\left(\int_{\Delta y_{\ell^{+} \ell^{-}}>0} d \sigma-\int_{\Delta y_{\ell^{+} \ell^{-}}<0} d \sigma\right),
$$

with $\Delta y_{\ell^{+} \ell^{-}}=y_{\ell^{+}}-y_{\ell^{-}}$. We present our results in table 5 , also reporting, for ease of comparisons, the results including UE and MPI and the ones for the uncorrelated case, which we dub PWG+HER' and PWG+PYT', where the (anti-)top-quark decay is performed by the SMC neglecting spin-correlation effects . Comparing the results including the spin correlation to the results where the top-quark decay is handled by the shower program - spin correlations are thus not taken into account in the latter predictions - we observe only a small difference between the two approaches. On the other hand a significant change can be observed when comparing LHEF results with the results including shower effects. Our conclusion is that most of the effect present at parton level is washed out by the parton shower. Indeed, investigating the behaviour of the leptonic forward-backward charge asymmetries in presence of a cut on the transverse momentum of the intermediate $t \bar{t}$ pair, one can trace back the large differences between results at partonic and hadronic level to the region of low $p_{\mathrm{T}}^{t \bar{t}}$ 's once again, as it was the case for the top-quark forward-backward charge asymmetry shown in table 3 .

\section{Conclusions}

We have studied top-quark hadroproduction in association with a jet merged with parton showers to NLO in QCD. For that purpose, we have presented an implementation of the process $t \bar{t}+$ jet in the framework of POWHEG BOX. A careful validation has been performed by comparing with known results. For Tevatron and LHC at $\sqrt{s}=7 \mathrm{TeV}$ we have investigated a large number of distributions, some of them with particular sensitivity to parton shower effects, such as the differential cross sections as function of the transverse momentum of the $t \bar{t}$-pair and the hardest jet. We have presented a detailed study of the impact of the parton-shower on the top-quark charge asymmetry. We find that the inclusion of the shower changes significantly the NLO predictions. A detailed analysis allowed us to trace back the origin of these corrections to the low $p_{T}^{t \bar{t}}$ region. We stress that the results presented here represent the first NLO evaluation of this distribution. Excluding the region $p_{T}^{t \bar{t}}<10 \mathrm{GeV}$ we find that the parton shower leads only to a marginal change of the charge asymmetry binned in $p_{T}^{t \bar{t}}$. A detailed comparison with future measurements may help in clarifying the discrepancy observed in the inclusive forward-backward charge asymmetry. However, we 
stress that in this work, we always refer to the asymmetries defined for the $t \bar{t} j$ sub-sample of the inclusive $t \bar{t}$ production.

We have also taken into account top-quark decays at LO in QCD, so that spin correlations, e.g., for the leptons from the decaying $t \bar{t}$-pair can be studied. Similar to the case of inclusive top-quark pair production the effects of spin correlations are clearly visible in double differential distributions of the top-quark (and anti-quark) decay products. Since $t \bar{t}+$ jet events represent an important fraction of all $t \bar{t}$ events, the measurement of this effect should be feasible at Tevatron and LHC.

The present work offers several options for extension. For instance, we have restricted ourselves in this article entirely to the study of top-quark hadroproduction, although the available QCD corrections at NLO [7, 8] are equally applicable to the process $b \bar{b}+$ jet with massive bottom-quarks. Therefore, the investigation of $b \bar{b}+$ jet in the framework of POWHEG BOX is feasible, even if further refinements in the code will be required to perform this task.

Most important, however, will be an extension of the phenomenological analysis presented in this paper to meet the real conditions of the experimental environment, e.g., studying more carefully tuning, UE and MPI effects of the SMC and, potentially, for other acceptance cuts. Also, results with different values of the top-quark mass, strong coupling constant $\alpha_{\mathrm{S}}\left(M_{Z}\right), \mu_{\mathrm{R}}, \mu_{\mathrm{F}}$ scales and with modern sets of PDFs will be needed.

Another natural development is the application of the merging procedure recently proposed in [73] to the MENLOPS improved $t \bar{t}$ and $t \bar{t} j$ samples, in order to improve the description of both fully inclusive $t \bar{t}$ and inclusive $t \bar{t}+$ jet productions. However, this will be the subject of a separate publication.

In general, we believe that a more thoroughly phenomenological study would be better performed in the framework of an experimental collaboration. Therefore, in order to facilitate all these future studies, the code of the POWHEG BOX implementation for the process $t \bar{t}+$ jet will be made publicly available on the web-page http://powhegbox.mib.infn.it.

\section{Acknowledgments}

We are thankful to M. Garzelli, E. Re and Z. Trocsanyi for useful discussions. This work has been supported by Helmholtz Gemeinschaft under contract VH-HA-101 (Alliance Physics at the Terascale), by Deutsche Forschungsgemeinschaft in Sonderforschungsbereich/Transregio 9 and by the European Commission through contract PITN-GA-2010264564 (LHCPhenoNet).

\section{A Folding}

In this appendix we briefly review the folding procedure, its implementation in the POWHEG BOX and the application to the present calculation. For a more detailed explanation of the procedure itself we refer to [32].

The POWHEG method allows for the generation of positive weighted events only. This is a direct consequence of the perturbative nature of the exclusive differential cross section integrated over the Born variables $\bar{B}$ (see e.g., eq. 4.2 in section 4 of [13] and comments 


\begin{tabular}{|ccccccc|}
\hline$\xi-y-\phi$ folding $\Longrightarrow$ & $1-1-1$ & $5-1-1$ & $1-5-1$ & $1-1-5$ & $5-5-5$ & $5-5-10$ \\
\hline TeV $1.96 \mathrm{TeV}, p_{\mathrm{T}}^{\text {gen }}=2 \mathrm{GeV}$ & 0.20 & 0.17 & 0.14 & 0.16 & 0.07 & 0.06 \\
$\mathrm{LHC} 7 \mathrm{TeV}, p_{\mathrm{T}}^{\text {gen }}=5 \mathrm{GeV}$ & 0.22 & 0.19 & 0.16 & 0.19 & 0.12 & 0.11 \\
$\mathrm{LHC} 14 \mathrm{TeV}, p_{\mathrm{T}}^{\text {gen }}=5 \mathrm{GeV}$ & 0.28 & 0.23 & 0.26 & 0.19 & 0.17 & 0.16 \\
\hline
\end{tabular}

Table 6. The fraction negative weighted events generated using different folding factors.

thereafter). In our implementations this integration is performed in the Monte Carlo approach: we generate fully exclusive configurations which depend also on the three variables associated to the extra radiation $(\xi, y, \phi$ in the FKS approach [26]) and then we sum over them discarding the extra dependency. However, the weight of this fully exclusive configurations, which is dubbed $\tilde{B}$, is not guaranteed to be always positive, in its enlarged phase space. The positivity of $\tilde{B}$ may always be recovered by keeping the Born variables fixed and redefining $\tilde{B}$ as the average over more phase space points, which differs only in the radiative variables. This clearly corresponds again to integrating over the radiative variables. This is the essence of the folding procedure: the number of phase space points considered in each evaluation of the $\tilde{B}$, for each radiative variable, is called folding factor.

An immediate drawback is that the folding procedure results in a increase of the program run time which is proportional to the product of the folding factors for the three radiation variables. In particular cases, it turns out to be more practical including negative weighted events in the final sample. However, these performance costs in the generation stage may be well balanced if full detector simulations are included or if it is required to have positive weights only. For this reasons, we report in table 6 the observed percent occurrence of negative weighted events in case of different folding levels and different colliders.

Open Access. This article is distributed under the terms of the Creative Commons Attribution Noncommercial License which permits any noncommercial use, distribution, and reproduction in any medium, provided the original author(s) and source are credited.

\section{References}

[1] P. Nason, S. Dawson and R. Ellis, The one particle inclusive differential cross-section for heavy quark production in hadronic collisions, Nucl. Phys. B 327 (1989) 49 [Erratum ibid. B 335 (1990) 260] [INSPIRE].

[2] W. Beenakker, W. van Neerven, R. Meng, G. Schuler and J. Smith, QCD corrections to heavy quark production in hadron hadron collisions, Nucl. Phys. B 351 (1991) 507 [INSPIRE].

[3] M.L. Mangano, P. Nason and G. Ridolfi, Heavy quark correlations in hadron collisions at next-to-leading order, Nucl. Phys. B 373 (1992) 295 [inSPIRE].

[4] M. Czakon and A. Mitov, Inclusive heavy flavor hadroproduction in NLO QCD: the exact analytic result, Nucl. Phys. B 824 (2010) 111 [arXiv:0811.4119] [INSPIRE].

[5] R. Bonciani, S. Catani, M.L. Mangano and P. Nason, NLL resummation of the heavy quark hadroproduction cross-section, Nucl. Phys. B 529 (1998) 424 [Erratum ibid. B 803 (2008) 234] [hep-ph/9801375] [INSPIRE]. 
[6] M. Aliev et al., HATHOR: HAdronic Top and Heavy quarks crOss section calculatoR, Comput. Phys. Commun. 182 (2011) 1034 [arXiv:1007.1327] [INSPIRE].

[7] S. Dittmaier, P. Uwer and S. Weinzierl, NLO QCD corrections to $t \bar{t}+$ jet production at hadron colliders, Phys. Rev. Lett. 98 (2007) 262002 [hep-ph/0703120] [INSPIRE].

[8] S. Dittmaier, P. Uwer and S. Weinzierl, Hadronic top-quark pair production in association with a hard jet at next-to-leading order QCD: phenomenological studies for the Tevatron and the LHC, Eur. Phys. J. C 59 (2009) 625 [arXiv:0810.0452] [INSPIRE].

[9] G. Bevilacqua, M. Czakon, C. Papadopoulos and M. Worek, Dominant QCD backgrounds in Higgs boson analyses at the LHC: a study of $p p \rightarrow t \bar{t}+2$ jets at next-to-leading order, Phys. Rev. Lett. 104 (2010) 162002 [arXiv:1002.4009] [INSPIRE].

[10] G. Bevilacqua, M. Czakon, C. Papadopoulos and M. Worek, Hadronic top-quark pair production in association with two jets at next-to-leading order QCD, Phys. Rev. D 84 (2011) 114017 [arXiv:1108.2851] [INSPIRE].

[11] S. Frixione and B.R. Webber, Matching NLO QCD computations and parton shower simulations, JHEP 06 (2002) 029 [hep-ph/0204244] [INSPIRE].

[12] P. Nason, A new method for combining NLO QCD with shower Monte Carlo algorithms, JHEP 11 (2004) 040 [hep-ph/0409146] [INSPIRE].

[13] S. Alioli, P. Nason, C. Oleari and E. Re, A general framework for implementing NLO calculations in shower Monte Carlo programs: the POWHEG BOX, JHEP 06 (2010) 043 [arXiv:1002.2581] [INSPIRE].

[14] R. Frederix et al., Scalar and pseudoscalar Higgs production in association with a top-antitop pair, Phys. Lett. B 701 (2011) 427 [arXiv:1104.5613] [INSPIRE].

[15] S. Frixione, P. Nason and B.R. Webber, Matching NLO QCD and parton showers in heavy flavor production, JHEP 08 (2003) 007 [hep-ph/0305252] [INSPIRE].

[16] S. Frixione, E. Laenen, P. Motylinski and B.R. Webber, Single-top production in MC@NLO, JHEP 03 (2006) 092 [hep-ph/0512250] [INSPIRE].

[17] S. Frixione, P. Nason and G. Ridolfi, A positive-weight next-to-leading-order Monte Carlo for heavy flavour hadroproduction, JHEP 09 (2007) 126 [arXiv:0707.3088] [INSPIRE].

[18] S. Alioli, P. Nason, C. Oleari and E. Re, NLO single-top production matched with shower in POWHEG: s- and t-channel contributions, JHEP 09 (2009) 111 [Erratum ibid. 1002 (2010) 011] [arXiv:0907.4076] [INSPIRE].

[19] E. Re, Single-top Wt-channel production matched with parton showers using the POWHEG method, Eur. Phys. J. C 71 (2011) 1547 [arXiv:1009.2450] [InSPIRE].

[20] K. Melnikov and M. Schulze, NLO QCD corrections to top quark pair production in association with one hard jet at hadron colliders, Nucl. Phys. B 840 (2010) 129 [arXiv: 1004.3284] [INSPIRE].

[21] G. Corcella et al., HERWIG 6: an event generator for hadron emission reactions with interfering gluons (including supersymmetric processes), JHEP 01 (2001) 010 [hep-ph/0011363] [INSPIRE].

[22] T. Sjöstrand, High-energy physics event generation with PYTHIA 5.7 and JETSET \%.4, Comput. Phys. Commun. 82 (1994) 74 [INSPIRE].

[23] T. Sjöstrand, S. Mrenna and P.Z. Skands, PYTHIA 6.4 physics and manual, JHEP 05 (2006) 026 [hep-ph/0603175] [INSPIRE]. 
[24] G. Bevilacqua et al., NLO QCD calculations with HELAC-NLO, Nucl. Phys. Proc. Suppl. 205-206 (2010) 211 [arXiv:1007.4918] [INSPIRE].

[25] A. Kardos, C. Papadopoulos and Z. Trócsányi, Top quark pair production in association with a jet with NLO parton showering, Phys. Lett. B 705 (2011) 76 [arXiv:1101.2672] [INSPIRE].

[26] S. Frixione, Z. Kunszt and A. Signer, Three jet cross-sections to next-to-leading order, Nucl. Phys. B 467 (1996) 399 [hep-ph/9512328] [INSPIRE].

[27] J. Alwall et al., MadGraph/MadEvent v4: the new web generation, JHEP 09 (2007) 028 [arXiv:0706.2334] [INSPIRE].

[28] K. Hasegawa, S. Moch and P. Uwer, AutoDipole: automated generation of dipole subtraction terms, Comput. Phys. Commun. 181 (2010) 1802 [arXiv:0911.4371] [INSPIRE].

[29] T. Binoth et al., A Proposal for a standard interface between Monte Carlo tools and one-loop programs, Comput. Phys. Commun. 181 (2010) 1612 [arXiv:1001.1307] [INSPIRE].

[30] G. van Oldenborgh and J. Vermaseren, New algorithms for one loop integrals, Z. Phys. C 46 (1990) 425 [InSPIRE].

[31] G. van Oldenborgh, FF: a package to evaluate one loop Feynman diagrams, Comput. Phys. Commun. 66 (1991) 1 [INSPIRE].

[32] S. Alioli, P. Nason, C. Oleari and E. Re, Vector boson plus one jet production in POWHEG, JHEP 01 (2011) 095 [arXiv: 1009.5594] [INSPIRE].

[33] J. Pumplin et al., New generation of parton distributions with uncertainties from global QCD analysis, JHEP 07 (2002) 012 [hep-ph/0201195] [INSPIRE].

[34] S.D. Ellis and D.E. Soper, Successive combination jet algorithm for hadron collisions, Phys. Rev. D 48 (1993) 3160 [hep-ph/9305266] [INSPIRE].

[35] S. Catani, S. Dittmaier, M.H. Seymour and Z. Trócsányi, THe dipole formalism for next-to-leading order QCD calculations with massive partons, Nucl. Phys. B 627 (2002) 189 [hep-ph/0201036] [INSPIRE].

[36] J. Alwall et al., A standard format for Les Houches event files, Comput. Phys. Commun. 176 (2007) 300 [hep-ph/0609017] [INSPIRE].

[37] M. Cacciari, G.P. Salam and G. Soyez, The anti-kt jet clustering algorithm, JHEP 04 (2008) 063 [arXiv:0802.1189] [INSPIRE].

[38] S. Alekhin, J. Blümlein, S. Klein and S. Moch, The 3, 4 and 5-flavor NNLO parton from deep-inelastic-scattering data and at hadron colliders, Phys. Rev. D 81 (2010) 014032 [arXiv:0908.2766] [INSPIRE].

[39] P. Jimenez-Delgado and E. Reya, Dynamical NNLO parton distributions, Phys. Rev. D 79 (2009) 074023 [arXiv:0810.4274] [InSPIRE].

[40] A. Martin, W. Stirling, R. Thorne and G. Watt, Parton distributions for the LHC, Eur. Phys. J. C 63 (2009) 189 [arXiv:0901.0002] [INSPIRE].

[41] G. Watt, Parton distribution function dependence of benchmark standard model total cross sections at the $7 \mathrm{TeV}$ LHC, JHEP 09 (2011) 069 [arXiv:1106.5788] [INSPIRE].

[42] U. Langenfeld, S. Moch and P. Uwer, Measuring the running top-quark mass, Phys. Rev. D 80 (2009) 054009 [arXiv:0906.5273] [INSPIRE].

[43] CDF collaboration, T. Aaltonen et al., First measurement of the t $\bar{t}$ differential cross section dsigma/d $M(t \bar{t})$ in $p \bar{p}$ collisions at $\sqrt{s}=1.96$ TeV, Phys. Rev. Lett. 102 (2009) 222003 [arXiv:0903.2850] [INSPIRE]. 


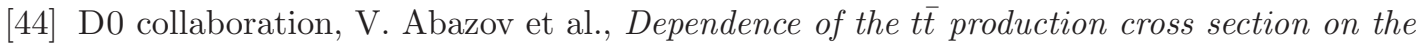
transverse momentum of the top quark, Phys. Lett. B 693 (2010) 515 [arXiv:1001.1900] [INSPIRE].

[45] D0 collaboration, V. Abazov et al., First measurement of the forward-backward charge asymmetry in top quark pair production, Phys. Rev. Lett. 100 (2008) 142002 [arXiv:0712.0851] [INSPIRE].

[46] CDF collaboration, T. Aaltonen et al., Forward-backward asymmetry in top quark production in $p \bar{p}$ collisions at $\sqrt{s}=1.96$ TeV, Phys. Rev. Lett. 101 (2008) 202001 [arXiv:0806.2472] [INSPIRE].

[47] CDF collaboration, T. Aaltonen et al., Evidence for a mass dependent forward-backward asymmetry in top quark pair production, Phys. Rev. D 83 (2011) 112003 [arXiv:1101.0034] [INSPIRE].

[48] D0 collaboration, V.M. Abazov et al., Forward-backward asymmetry in top quark-antiquark production, Phys. Rev. D 84 (2011) 112005 [arXiv:1107.4995] [INSPIRE].

[49] S. Catani and B. Webber, Infrared safe but infinite: soft gluon divergences inside the physical region, JHEP 10 (1997) 005 [hep-ph/9710333] [INSPIRE].

[50] S. Alioli, K. Hamilton, P. Nason, C. Oleari and E. Re, Jet pair production in POWHEG, JHEP 04 (2011) 081 [arXiv: 1012.3380] [INSPIRE].

[51] P.Z. Skands, Tuning monte carlo generators: the Perugia tunes, Phys. Rev. D 82 (2010) 074018 [arXiv: 1005.3457] [InSPIRE].

[52] V. Ahrens, A. Ferroglia, M. Neubert, B.D. Pecjak and L.L. Yang, The top-pair forward-backward asymmetry beyond NLO, Phys. Rev. D 84 (2011) 074004 [arXiv:1106.6051] [INSPIRE].

[53] J.H. Kühn and G. Rodrigo, Charge asymmetries of top quarks at hadron colliders revisited, arXiv: 1109.6830 [INSPIRE].

[54] S. Alioli et al., A theoretical study of new observables to measure the top quark mass at hadroncolliders, in Lepton Photon 2011, August 22-27, Tata Institute of Fundamental Research, Mumbai, India (2011), http://www.ino.tifr.res.in/MaKaC/contributionDisplay.py?contribId=24\&confId=79.

[55] ATLAS collaboration, Measurement of the charge asymmetry in top quark pair production in pp collisions at $\sqrt{s}=7$ TeV using the ATLAS detector, ATLAS-CONF-2011-106 (2011).

[56] CMS collaboration, Measurement of the charge asymmetry in top quark pair production, PAS-TOP-11-014 (Measurement of the Charge Asymmetry in Top Quark Pair Production).

[57] A. Denner, S. Dittmaier, S. Kallweit and S. Pozzorini, NLO QCD corrections to WWbb production at hadron colliders, Phys. Rev. Lett. 106 (2011) 052001 [arXiv:1012.3975] [INSPIRE].

[58] G. Bevilacqua, M. Czakon, A. van Hameren, C.G. Papadopoulos and M. Worek, Complete off-shell effects in top quark pair hadroproduction with leptonic decay at next-to-leading order, JHEP 02 (2011) 083 [arXiv: 1012.4230] [INSPIRE].

[59] W. Bernreuther, A. Brandenburg, Z. Si and P. Uwer, Top quark spin correlations at hadron colliders: predictions at next-to-leading order QCD, Phys. Rev. Lett. 87 (2001) 242002 [hep-ph/0107086] [inSPIRE]. 
[60] A. Brandenburg, Z. Si and P. Uwer, QCD corrected spin analyzing power of jets in decays of polarized top quarks, Phys. Lett. B 539 (2002) 235 [hep-ph/0205023] [INSPIRE].

[61] S. Frixione, E. Laenen, P. Motylinski and B.R. Webber, Angular correlations of lepton pairs from vector boson and top quark decays in Monte Carlo simulations, JHEP 04 (2007) 081 [hep-ph/0702198] [INSPIRE].

[62] C. Oleari and L. Reina, $W^{ \pm} b \bar{b}$ production in POWHEG, JHEP 08 (2011) 061 [Erratum ibid. 1111 (2011) 040] [arXiv:1105.4488] [InSPIRE].

[63] S. Frixione, E. Laenen, P. Motylinski, B.R. Webber and C.D. White, Single-top hadroproduction in association with a W boson, JHEP 07 (2008) 029 [arXiv:0805.3067] [INSPIRE].

[64] G. Mahlon and S.J. Parke, Spin correlation effects in top quark pair production at the LHC, Phys. Rev. D 81 (2010) 074024 [arXiv: 1001.3422] [INSPIRE].

[65] P. Uwer, Maximizing the spin correlation of top quark pairs produced at the Large Hadron Collider, Phys. Lett. B 609 (2005) 271 [hep-ph/0412097] [INSPIRE].

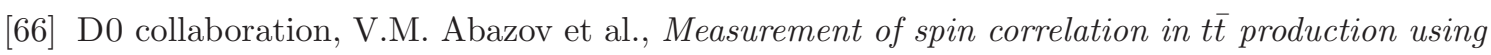
dilepton final states, Phys. Lett. B 702 (2011) 16 [arXiv:1103.1871] [INSPIRE].

[67] K. Melnikov and M. Schulze, Top quark spin correlations at the Tevatron and the LHC, Phys. Lett. B 700 (2011) 17 [arXiv:1103.2122] [INSPIRE].

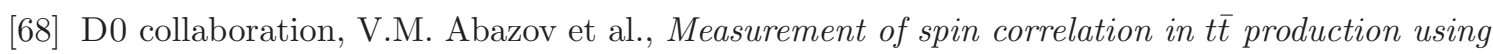
a matrix element approach, Phys. Rev. Lett. 107 (2011) 032001 [arXiv:1104.5194] [INSPIRE].

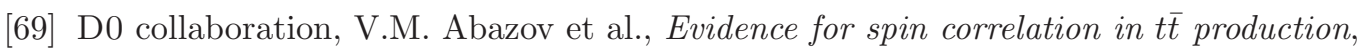
submitted to Phys. Rev. Lett. (2011), arXiv:1110.4194 [INSPIRE].

[70] M.L. Mangano, M. Moretti, F. Piccinini and M. Treccani, Matching matrix elements and shower evolution for top-quark production in hadronic collisions, JHEP 01 (2007) 013 [hep-ph/0611129] [INSPIRE].

[71] ATLAS collaboration, Measurement of spin correlation in t $t \bar{t}$ production from pp collisions at $\sqrt{s}=7 \mathrm{TeV}$ using the ATLAS detector, ATLAS-CONF-2011-117 (2011).

[72] W. Bernreuther and Z.-G. Si, Distributions and correlations for top quark pair production and decay at the Tevatron and LHC., Nucl. Phys. B 837 (2010) 90 [arXiv:1003.3926] [INSPIRE].

[73] S. Alioli, K. Hamilton and E. Re, Practical improvements and merging of POWHEG simulations for vector boson production, JHEP 09 (2011) 104 [arXiv:1108.0909] [INSPIRE]. 